MATHEMATICS OF COMPUTATION

Volume 73 , Number 246 , Pages 659-689

S 0025-5718(03)01550-3

Article electronically published on October 17, 2003

\title{
SUBSTRUCTURING PRECONDITIONERS FOR THE THREE FIELDS DOMAIN DECOMPOSITION METHOD
}

\author{
SILVIA BERTOLUZZA
}

\begin{abstract}
We study a class of preconditioners based on substructuring, for the discrete Steklov-Poincaré operator arising in the three fields formulation of domain decomposition in two dimensions. Under extremely general assumptions on the discretization spaces involved, an upper bound is provided on the condition number of the preconditioned system, which is shown to grow at most as $\log (H / h)^{2}(H$ and $h$ denoting, respectively, the diameter and the discretization mesh-size of the subdomains). Extensive numerical tests-performed on both a plain and a stabilized version of the method-confirm the optimality of such bound.
\end{abstract}

\section{INTRODUCTION}

The use of nonconforming domain decomposition methods is becoming increasingly popular. This is due to several factors: the possibility of easily coupling different discretizations in different subdomains without the need for imposing strong matching conditions allows us to employ without any adaptation the technologies developed for treating the problem with a single-domain approach. As an example, in an adaptive strategy, refinement can be carried out in each subdomain independently. Moreover, it is possible to use a different type of discretization (finite elements, spectral methods, wavelets) in the different subdomains, and therefore to employ, in each subdomain, the best-suited method (spectral methods where the solution is expected to be very smooth, finite elements where a complicated geometry requires it, wavelets where isolated singularities in a regular background are expected).

Among the different nonconforming domain decomposition method, we consider here the three fields formulation, as proposed by F. Brezzi and L.D. Marini in [14]. Differently from other nonconforming formulations 3 , 18 of domain decomposition, weak continuity is not imposed by requiring that the jump across the interface be orthogonal to some multiplier space, but by introducing a space $\Phi_{h}$, approximating the traces of functions in $H^{1}(\Omega)$ on the interface $\Sigma$. The elements of $\Phi_{h}$ are to be used as a Dirichlet boundary condition — imposed by means of Lagrange multipliers - for the functions in the subdomains. As with other nonconforming methods, great freedom is left in the choice of the discretization in the subdomains which,

Received by the editor November 6, 2000 and, in revised form, February 22, 2002.

2000 Mathematics Subject Classification. Primary 65N55, 65N22.

Key words and phrases. Three fields formulation, domain decomposition, stabilized methods, preconditioning. 
after applying a suitable stabilization method if needed [2, 7], can be chosen independently from each other. The disadvantage of introducing a third unknown field is compensated for by the fact that all subdomains are treated exactly in the same way, which results in an easier implementation and possibly, when considering the parallelization of the method, in an easier balancing of the load between processors.

We deal, in this paper, with the problem of the efficient solution of the linear system arising when applying such a method. By applying a Schur complement technique, the solution of the resulting discrete problem can be reduced to the solution of an equation on the "trace" unknown, involving a Steklov-Poincaré type operator which can be shown to be a pseudo-differential operator of order one. The resulting linear system will then be solved by an iterative procedure, at each step of which there will be the need of computing the action of such operator on a given function. This reduces to solving independent Dirichlet problems on the subdomains, a task which can be carried out in parallel, subdomain by subdomain in a completely independent way.

In order to have an efficient scheme for solving the equation for the interface unknown, we will then need two things:

- efficient solvers for the local Dirichlet problems on the subdomains;

- a preconditioner for the discrete Steklov-Poincaré operator so that the number of iterations is kept as low as possible.

We will concentrate here on the second issue, by analyzing and testing a class of preconditioners for the discrete Steklov-Poincaré operator. The approach that we will follow is the substructuring one, proposed by J.H. Bramble, J.E. Pasciak and A.H. Schatz [9] in the framework of conforming domain decomposition and already applied in the framework of nonconforming methods to the mortar method in 1. This consists in decomposing the trace space $\Phi_{h}$ in a direct sum of a coarse subspace (essentially linked to the geometrical decomposition of $\Omega$ in subdomains) and of suitable local subspaces and then considering the related block-Jacobi type preconditioners.

From the theoretical point of view, we study the dependence of the condition number of the resulting preconditioned matrix on the mesh size of the discretizations, aiming at giving bounds which, under suitable assumptions, are independent of the number and size of the subdomains. In particular we provide an upper bound which applies to a wide variety of approximation spaces, including finite elements and wavelets. Though only suboptimal in terms of the mesh size on the skeleton (the condition number of the preconditioned matrix grows like $(\log (H / h))^{2}$, $H$ and $h$ being, respectively, the diameter of the subdomains and the mesh size of the corresponding discretization), the estimate obtained is uniform with respect to the number and size of subdomains. The resulting method is then potentially almost perfectly scalable. Moreover this approach has the advantage over other asymptotically better preconditioners for the three fields formulation ([7]) of being itself naturally computable in parallel (each edge in the skeleton being treated independently of the others).

We conclude the paper by reporting a series of extensive numerical tests, performed on both the plain and on a stabilized formulation as proposed in [7]. The results of the tests are in agreement with the theory and confirm the polylogarithmic growth (with respect to the mesh size of the discretization) of the preconditioned 
system, as well as the independence (asymptotically) with respect to the number and size of the subdomains.

\section{Domain DECOMPOSITION METHOD: THE THREE FIELDS FORMULATION}

Let $\Omega \subset \mathbb{R}^{2}$ be a convex polygonal domain. We will consider the following simple model problem: given $f \in L^{2}(\Omega)$, find $u$ satisfying

$$
-\sum_{i, j=1}^{2} \frac{\partial}{\partial \mathbf{x}_{j}}\left(a_{i, j}(\mathbf{x}) \frac{\partial u}{\partial \mathbf{x}_{i}}\right)+a_{0}(\mathbf{x}) u=f \text { in } \Omega, \quad u=0 \text { on } \partial \Omega .
$$

We assume that for almost all $\mathbf{x} \in \Omega$ we have $0 \leq a_{0}(\mathbf{x}) \leq R$ and that the matrix $\left(a_{i j}(\mathbf{x})\right)_{i, j=1,2}$ is symmetric positive definite, with smallest eigenvalue $\geq \alpha>0$ and largest eigenvalue $\leq \alpha^{\prime}, \alpha, \alpha^{\prime}$ independent of $\mathbf{x}$.

In a domain decomposition framework, we will consider the three fields formulation ([14]) of such a problem. Let $\bar{\Omega}=\bigcup_{k=1}^{K} \bar{\Omega}_{k}, \Omega_{k}$ polygons, which, for simplicity, we will assume to be quadrangles. We set $\Gamma_{k}=\partial \Omega_{k}, \Sigma=\bigcup_{k} \Gamma_{k}$ being the skeleton of the decomposition. Remark that differently from [14] here we define $\Sigma$ so that it includes the external boundary of the domain $\Omega$, so that there is no need of distinguishing between interior subdomains and subdomains which are adjacent to the boundary $\partial \Omega$. This results in a simplification both on the notational and on the implementation point of view, since all subdomains are treated in the same way. We will make the following regularity assumptions on the subdomains $\Omega_{k}$ :

(A1) The subdomains are regular in shape and the geometrical decomposition is graded, that is,

(a) there exists a positive constant $c_{0}$ such that, for all $k, \Omega_{k}$ contains a ball of diameter $c_{0} H_{k}$, it is contained in a ball of diameter $H_{k}$, and the length of each side is bounded from below by $c_{0} H_{k}$; moreover any interior angle $\omega$ satisfies $0<c_{1}<\omega<c_{2}<\pi\left(c_{0}, c_{1}\right.$, and $c_{2}$ independent of $k$ );

(b) there exists a positive constant $c_{3}$ such that, if $\ell, k$ are such that $\left|\partial \Omega_{k} \cap \partial \Omega_{\ell}\right|>0$, then it holds that

$$
H_{k} / H_{\ell} \leq c_{3}
$$

For technical reasons, we also need to make the following assumption (needed for the proof of (25) in the following), which in many cases can be shown to be actually a consequence of the previous one:

(A2) There exists a constant $C_{0}$ such that for all $y$, denoting by $\ell_{y}$ the line of equation $\mathbf{x}_{2}=y$ and setting $K_{y}=\left\{k: \operatorname{length}\left(\ell_{y} \cap \Omega_{k}\right)>0\right\}$, it holds that $\sum_{k \in K_{y}} H_{k} \leq C_{0}$.

We are interested here in explicitly studying the dependence of the estimates that we are going to prove on the number and size of the subdomains. To this end, in the following we will employ the notation $A \lesssim B$ (resp. $A \gtrsim B$ ) to say that the quantity $A$ is bounded from above (resp. from below) by $c B$, with a constant $c$ independent of $k$ and of the $H_{k}$ 's, as well as of any mesh size parameter. The expression $A \simeq B$ will stand for $A \lesssim B \lesssim A$.

For each $k$ we let the norm and seminorm of $H^{1}\left(\Omega_{k}\right)$ be defined as usual by $\|u\|_{H^{1}\left(\Omega_{k}\right)}^{2}=\int_{\Omega_{k}}|u|^{2} d \mathbf{x}+\int_{\Omega_{k}}|\nabla u|^{2} d \mathbf{x}$ and $|u|_{H^{1}\left(\Omega_{k}\right)}^{2}=\int_{\Omega_{k}}|\nabla u|^{2} d \mathbf{x}$. On the 
boundary $\partial \Omega_{k}$ we let the norm of $H^{1 / 2}\left(\partial \Omega_{k}\right)$ be defined by

$$
\|\varphi\|_{H^{1 / 2}\left(\partial \Omega_{k}\right)}=\inf _{\substack{u \in H^{1}\left(\Omega_{k}\right) \\ u=\varphi \text { on } \partial \Omega_{k}}}\|u\|_{H^{1}\left(\Omega_{k}\right)} .
$$

For all $s$ in $] 0,1\left[\right.$, we define the $H^{s}\left(\partial \Omega_{k}\right)$ seminorm by

$$
|\varphi|_{H^{s}\left(\partial \Omega_{k}\right)}^{2}=\int_{\partial \Omega_{k}} \int_{\partial \Omega_{k}} \frac{(\varphi(x)-\varphi(y))^{2}}{|x-y|^{2 s+1}} d s(x) d s(y) .
$$

The space $H^{-1 / 2}\left(\partial \Omega_{k}\right)$ is defined as the dual of $H^{1 / 2}\left(\partial \Omega_{k}\right)$.

For $e \subset \partial \Omega_{k}$ segment of extrema $a$ and $b$, we define the $H^{s}(e)$ seminorm as usual by the integral expression (2) where the integrals are taken over $e$. On $e$ we will also consider the spaces $H_{0}^{s}(e)(s \neq 1 / 2)$ and $H_{00}^{1 / 2}(e)$ of functions whose extension by zero is in $H^{s}\left(\partial \Omega_{k}\right)(s \neq 1 / 2)$ and $H^{1 / 2}\left(\partial \Omega_{k}\right)$, respectively, which we will equip with the norm

$$
\|\varphi\|_{H_{00}^{1 / 2}(e)}^{2}=|\varphi|_{H^{1 / 2}(e)}^{2}+\int_{e} \frac{|\varphi(x)|^{2}}{|x-a|^{2 s}} d s(x)+\int_{e} \frac{|\varphi(x)|^{2}}{|x-b|^{2 s}} d s(x) .
$$

We recall that for $s<1 / 2$ the two spaces $H^{s}(e)$ and $H_{0}^{s}(e)$ coincide, and the two corresponding norms are equivalent. However, the constant in the equivalence depends a priori on $H_{k}$ and on $s$. In particular it explodes as $s$ converges to $1 / 2$. The behaviour of such a constant as $s$ approaches the limit value $1 / 2$ (see (44)) will play a key role in the forthcoming analysis.

Remark also that, for $\varphi \in H_{00}^{1 / 2}(e)$, letting $\check{\varphi} \in H^{1 / 2}\left(\partial \Omega_{k}\right)$ denote the function coinciding with $\varphi$ on $e$ and identically vanishing on $\partial \Omega_{k} \backslash e$, it holds that

$$
\|\varphi\|_{H_{00}^{1 / 2}(e)} \simeq|\varphi|_{H^{1 / 2}\left(\partial \Omega_{k}\right)},
$$

the constant in the equivalence being uniformly bounded, provided that the ratio between the length of $\partial \Omega_{k}$ and the length of $e$ is uniformly bounded.

The functional setting for the three fields domain decomposition method is given by the following spaces:

$$
V=\prod_{k=1}^{K} H^{1}\left(\Omega_{k}\right), \quad \Lambda=\prod_{k=1}^{K} H^{-1 / 2}\left(\partial \Omega_{k}\right),
$$

and

$$
\Phi=\left\{\varphi \in L^{2}(\Sigma) \text { : there exists } u \in H_{0}^{1}(\Omega), u=\varphi \text { on } \Sigma\right\}=\left.H_{0}^{1}(\Omega)\right|_{\Sigma},
$$

respectively equipped with the norms:

$$
\|u\|_{V}=\left(\sum_{k}\left\|u^{k}\right\|_{H^{1}\left(\Omega_{k}\right)}^{2}\right)^{1 / 2}, \quad\|\lambda\|_{\Lambda}=\left(\sum_{k}\left\|\lambda^{k}\right\|_{H^{-1 / 2}\left(\partial \Omega_{k}\right)}^{2}\right)^{1 / 2},
$$

and (see [4])

$$
\|\varphi\|_{\varphi}=\inf _{\substack{u \in H_{0}^{1}(\Omega) \\ u=\varphi \text { on } \Sigma}}\|u\|_{H^{1}(\Omega)} \simeq\left(\sum_{k}|\varphi|_{H^{1 / 2}\left(\partial \Omega_{k}\right)}^{2}\right)^{1 / 2} .
$$


Let $a^{k}: H^{1}\left(\Omega_{k}\right) \times H^{1}\left(\Omega_{k}\right) \rightarrow \mathbb{R}$ denote the bilinear form corresponding to the linear operator considered:

$$
a^{k}(w, v)=\int_{\Omega_{k}}\left(\sum_{i, j=1}^{2} a_{i j}(\mathbf{x}) \frac{\partial w}{\partial \mathbf{x}_{i}} \frac{\partial v}{\partial \mathbf{x}_{j}}+a_{0}(\mathbf{x}) w v\right) d \mathbf{x} .
$$

Under the assumptions made on the matrix $\left(a_{i j}(\mathbf{x})\right)_{i, j=1,2}$ and on $a_{0}$, the bilinear forms $a^{k}$ are uniformly $H^{1}\left(\Omega_{k}\right)$-continuous, and semidefinite. More precisely, there exist positive constants $\alpha$ and $L$ independent of $k$ and $H_{k}$ such that for all $w, v$ in $H^{1}\left(\Omega_{k}\right)$

$$
\alpha|w|_{H^{1}\left(\Omega_{k}\right)}^{2} \leq a^{k}(w, w), \quad\left|a^{k}(w, v)\right| \leq L\|w\|_{H^{1}\left(\Omega_{k}\right)}\|v\|_{H^{1}\left(\Omega_{k}\right)} .
$$

The three fields formulation of equation (1) is the following: find $(u, \lambda, \varphi) \in V \times$ $\Lambda \times \Phi$ such that

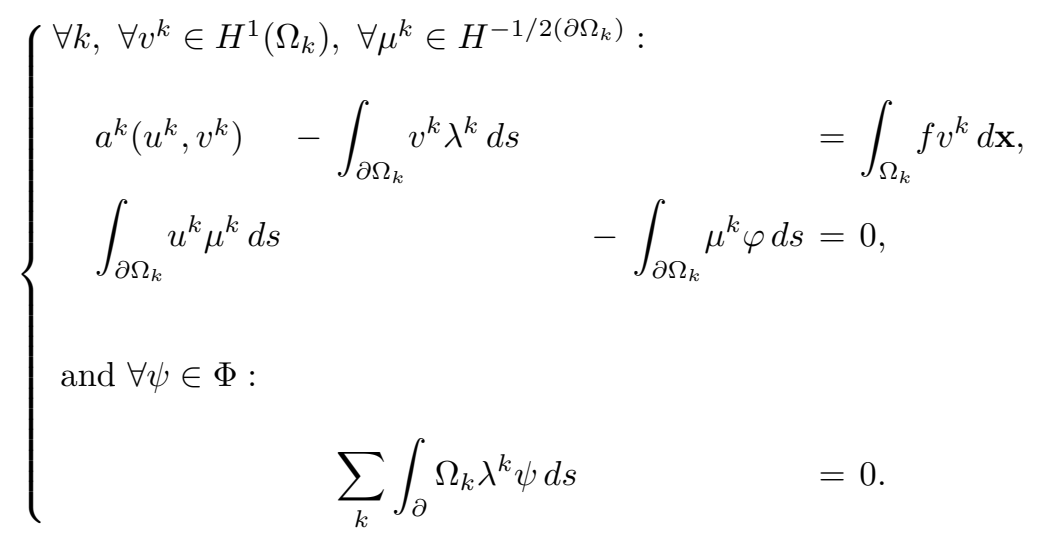

It is known that this problem admits a unique solution $(u, \lambda, \varphi)$ where $u$ is indeed the solution of (1) and such that

$$
\lambda^{k}=\frac{\partial u^{k}}{\partial \nu_{a}^{k}} \text { on } \Gamma_{k}, \quad \varphi=u \text { on } \Sigma
$$

where $\nu_{a}^{k}$ denotes the outer conormal derivative to the subdomain $\Omega_{k}$. Remark that in such a formulation the homogeneous Dirichlet boundary condition is embedded in the definition of the space $\Phi$.

Equation (8) can be discretized by a Galerkin scheme: after choosing discretization spaces $V_{h}=\prod_{k=1}^{K} V_{h}^{k} \subset \prod_{k=1}^{K} H^{1}\left(\Omega_{k}\right), \Lambda_{h}=\prod_{k=1}^{K} \Lambda_{h}^{k} \subset \prod_{k=1}^{K} H^{-1 / 2}\left(\partial \Omega_{k}\right)$ and $\Phi_{h} \subset \Phi$, we consider the problem: find $\left(u_{h}, \lambda_{h}, \varphi_{h}\right) \in V_{h} \times \Lambda_{h} \times \Phi_{h}$, such that 
one has

$$
\begin{cases}\forall k, \forall v_{h}^{k} \in V_{h}^{k}, \forall \mu_{h}^{k} \in \Lambda_{h}^{k}: & =\int_{\Omega_{k}} f v_{h}^{k} d \mathbf{x}, \\ a^{k}\left(u_{h}^{k}, v_{h}^{k}\right) \quad-\int_{\partial \Omega_{k}} v_{h}^{k} \lambda_{h}^{k} d s & -\int_{\partial \Omega_{k}} \mu_{h}^{k} \varphi_{h} d s=0, \\ \int_{\partial \Omega_{k}} u_{h}^{k} \mu_{h}^{k} d s & =0 . \\ \text { and } \forall \psi_{h} \in \Phi_{h}: & \\ \sum_{k} \int_{\partial} \Omega_{k} \lambda_{h}^{k} \psi_{h} d s & =0\end{cases}
$$

Existence, uniqueness and stability of the solution of (10) rely on the validity of suitable inf-sup conditions. More precisely we can make the following assumption:

(A3) The spaces $V_{h}^{k}, \Lambda_{h}^{k}$ and $\Phi_{h}$ are such that

(a) $\Lambda_{h}^{k}$ contains the constant functions;

(b) the following compatibility condition between $V_{h}^{k}$ and $\Lambda_{h}^{k}$ holds uniformly in $k$ :

$$
\inf _{\lambda_{h}^{k} \in \Lambda_{h}^{k}} \sup _{u_{h}^{k} \in V_{h}^{k}} \frac{\int_{\partial} \Omega_{k} \lambda_{h}^{k} u_{h}^{k} d s}{\left\|u_{h}^{k}\right\|_{H^{1}\left(\Omega_{k}\right)}\left\|\lambda_{h}^{k}\right\|_{H^{-1 / 2}\left(\partial \Omega_{k}\right)}} \geq \beta>0 ;
$$

(c) the following compatibility condition between $\Lambda_{h}$ and $\Phi_{h}$ holds:

$$
\inf _{\varphi_{h} \in \Phi_{h}} \sup _{\lambda_{h} \in \Lambda_{h}} \frac{\sum_{k} \int_{\partial} \Omega_{k} \lambda_{h}^{k} \varphi_{h} d s}{\left\|\lambda_{h}\right\|_{\Lambda}\left\|\varphi_{h}\right\|_{\Phi}} \geq \alpha>0 .
$$

Remark that (A3)(a) implies that for all $v_{h}^{k} \in \operatorname{ker} B_{h}^{k}=\left\{v_{h}^{k} \in V_{h}^{k}: \int_{\partial} \Omega_{k} v_{h}^{k} \mu_{h}^{k}=\right.$ $\left.0 \forall \mu_{h}^{k} \in \Lambda_{h}^{k}\right\}$ it holds that $\int_{\partial} \Omega_{k} v_{h}^{k} d s=0$ and then it is not difficult to see that $a$ is elliptic on $\operatorname{ker} B_{h}^{k}$. Then, by a well-known argument ([11]), there exists a unique solution to problem (10), which converges with an optimal rate to the solution of (8).

The linear system stemming from (10) takes the form

$$
\left(\begin{array}{ccc}
A & B^{T} & 0 \\
B & 0 & C^{T} \\
0 & C & 0
\end{array}\right) \cdot\left(\begin{array}{c}
\underline{u}_{h} \\
\underline{\lambda}_{h} \\
\underline{\varphi}_{h}
\end{array}\right)=\left(\begin{array}{c}
\underline{f}_{h} \\
0 \\
0
\end{array}\right),
$$

$\left(\underline{u}_{h}, \underline{\lambda}_{h}\right.$, and $\underline{\varphi}_{h}$ being the vectors of the coefficients of $u_{h}, \lambda_{h}$ and $\varphi_{h}$ in the bases chosen for $V_{h}, \Lambda_{h}$ and $\Phi_{h}$, respectively). By a Schur complement argument the solution of (13) can be reduced to a system in the unknown $\underline{\varphi}_{h}$ of the form

$$
\mathbb{C A}^{-1} \mathbb{C}^{T} \underline{\varphi}_{h}=\mathbb{C A}^{-1}\left(\begin{array}{c}
f_{h} \\
0
\end{array}\right), \quad \mathbb{C}=\left[\begin{array}{ll}
0 & C
\end{array}\right], \quad \mathbb{A}=\left(\begin{array}{cc}
A & B^{T} \\
B & 0
\end{array}\right) .
$$

The matrix $S=\mathbb{C A}^{-1} \mathbb{C}^{T}$ does not need to be assembled. The system (14) can rather be solved by an iterative technique (like for instance a conjugate gradient 
method) and therefore only the action of $S$ on a given vector needs to be implemented. Multiplying by $S$ implies the need for solving a linear system with matrix $\mathbb{A}$. This reduces, by a proper reordering of the unknowns, to independently solving $K$ discrete Dirichlet problems with Lagrange multipliers in the $K$ subdomains. Moreover under the above assumptions it is possible to prove that the bilinear form $s: \Phi_{h} \times \Phi_{h} \longrightarrow \mathbb{R}$,

$$
s\left(\varphi_{h}, \psi_{h}\right)=\underline{\psi}_{h}^{T} S \underline{\varphi}_{h}, \quad S=\mathbb{C A}^{-1} \mathbb{C}^{T},
$$

corresponding to the Schur complement matrix $S$, satisfies the following continuity and coercivity assumptions:

Lemma 2.1. If (A3) holds, then the bilinear form $s$ is continuous and coercive with respect to the $\Phi$ norm:

$$
\begin{aligned}
& s\left(\varphi_{h}, \psi_{h}\right) \lesssim\left\|\varphi_{h}\right\|_{\Phi}\left\|\psi_{h}\right\|_{\Phi}, \\
& s\left(\varphi_{h}, \varphi_{h}\right) \gtrsim\left\|\varphi_{h}\right\|_{\Phi}^{2} .
\end{aligned}
$$

Assumption (A3) is, in practice, quite restrictive. In particular the requirement that both (A3)(b) and (A3)(c) hold simultaneously poses some serious limitation on the choice of the discretization spaces for the three unknowns $u, \lambda$ and $\varphi$. In order to be more free in such a choice, and more specifically in order to be allowed to choose the discretization space for the interface unknown $\varphi$ independently of the choice of the other two discretization spaces, it is also possible to consider a stabilized version. Different ways have been proposed for stabilizing the three fields formulation $([2,12,13])$. We consider here the approach of [7, 4]. For all $k$ let a bilinear form $[\cdot, \cdot]_{\frac{1}{2}, k}: H^{1 / 2}\left(\partial \Omega_{k}\right) \times H^{1 / 2}\left(\partial \Omega_{k}\right) \rightarrow \mathbb{R}$ be given, satisfying the following bounds: for all $\eta, \xi \in H^{1 / 2}\left(\partial \Omega_{k}\right)$

$$
[\eta, \eta]_{\frac{1}{2}, k} \geq 0, \quad[\eta, \xi]_{\frac{1}{2}, k} \leq B|\eta|_{1 / 2, \partial \Omega_{k}}|\xi|_{1 / 2, \partial \Omega_{k}},
$$

for $B$ positive constant independent of $k$ and $H_{k}$.

We consider then the following discrete stabilized formulation: find $u_{h} \in V_{h}$, $\lambda_{h} \in \Lambda_{h}, \varphi_{h} \in \Phi_{h}$ such that it holds that

(19)

$$
\left\{\begin{array}{l}
\forall k, \forall v_{h}^{k} \in V_{h}^{k}, \forall \mu_{h}^{k} \in \Lambda_{h}^{k}: \\
a^{k}\left(u_{h}^{k}, v_{h}^{k}\right)+\gamma\left[u_{h}^{k}, v_{h}^{k}\right]_{\frac{1}{2}, k}-\int_{\partial \Omega_{k}} v_{h}^{k} \lambda_{h}^{k} d s-\gamma\left[\varphi_{h}, v_{h}^{k}\right]_{\frac{1}{2}, k}=\int_{\Omega_{k}} f v_{h}^{k} d \mathbf{x}, \\
\int_{\partial \Omega_{k}} u_{h}^{k} \mu_{h}^{k} d s \\
\text { and } \forall \psi_{h} \in \Phi_{h}: \\
-\sum_{k} \gamma\left[u_{h}^{k}, \psi_{h}^{k}\right]_{\frac{1}{2}, k}+\sum_{k} \int_{\partial} \Omega_{k} \lambda_{h}^{k} \psi_{h} d s+\sum_{k} \gamma\left[\varphi_{h}, \psi_{h}\right]_{\frac{1}{2}, k}=0,
\end{array}\right.
$$

where $\gamma>0$ is a parameter independent of the choice of the discretization spaces. Such formulation is consistent with the original continuous problem, i.e., by substituting the solution $(u, \lambda, \varphi)$ of (8) at the place of $\left(u_{h}, \lambda_{h}, \varphi_{h}\right)$ in (19), we obtain an identity.

The assumptions needed to guarantee existence and uniqueness of the solution of the discrete problem, as well as an error estimate, can now be made on the one 
hand on the spaces $V_{h}$ and $\Lambda_{h}$ and on the other hand in a completely independent way, on the action of the bilinear forms $[\cdot, \cdot]_{\frac{1}{2}, k}$ on $\left.\Phi_{h}\right|_{\partial} \Omega_{k}$. For example we can replace assumption $(\mathrm{A} 3)(\mathrm{c})$ with

(A3)(c') There exists a positive constant $b$ such that for all $\varphi_{h} \in \Phi_{h}$ it holds for all $k$ that

$$
\left[\varphi_{h}, \varphi_{h}\right]_{\frac{1}{2}, k} \geq b\left|\varphi_{h}\right|_{H^{1 / 2}\left(\partial \Omega_{k}\right)}^{2} .
$$

It is then possible to show that the discrete problem (19) admits a unique solution satisfying an optimal error estimate (4]). It is beyond the goals of this paper to thoroughly discuss the construction of suitable bilinear forms $[\cdot, \cdot]_{\frac{1}{2}, k}$. We recall that this can be done with the aid of multiscale techniques and we refer to 7, 5, 10, 6] for a detailed analysis of possible solutions.

The linear system stemming from such a problem takes the following form this time:

$$
\left(\begin{array}{ccc}
\check{A} & B^{T} & -\gamma D^{T} \\
B & 0 & C^{T} \\
-\gamma D & C & \gamma E
\end{array}\right) \cdot\left(\begin{array}{c}
\underline{u}_{h} \\
\underline{\lambda}_{h} \\
\underline{\varphi}_{h}
\end{array}\right)=\left(\begin{array}{c}
f \\
\underline{f}_{h} \\
0 \\
0
\end{array}\right),
$$

with $\breve{A}=A+\gamma F$ (the matrices $D, E$ and $F$ deriving from the stabilizing terms).

Again, the solution of (20) can be reduced to a system in the unknown $\underline{\varphi}_{h}$, this time taking the form

$$
S \underline{\varphi}_{h}:=\left(-\mathbb{D} \check{A}^{-1} \mathbb{D}^{T}+\gamma E\right) \underline{\varphi}_{h}=-\mathbb{D} \mathbb{A}^{-1}\left(\begin{array}{c}
f_{h} \\
0
\end{array}\right)
$$

with

$$
\check{\mathbb{A}}=\left(\begin{array}{cc}
\check{A} & B^{T} \\
B & 0
\end{array}\right), \quad \mathbb{D}=\left[\begin{array}{ll}
-\gamma D & C
\end{array}\right] .
$$

Once again we let $s: \Phi_{h} \times \Phi_{h} \longrightarrow \mathbb{R}$ be the bilinear form corresponding to the Schur complement matrix $S$

$$
s\left(\varphi_{h}, \psi_{h}\right)=\underline{\psi}_{h}^{T} S \underline{\varphi}_{h},
$$

and also for the stabilized formulation the following holds (4]):

Lemma 2.2. Under assumptions $(\mathrm{A} 3)(\mathrm{a}),(\mathrm{b}),\left(\mathrm{c}^{\prime}\right)$ the bilinear form $s$ is continuous and coercive with respect to the $\Phi$ norm:

$$
\begin{aligned}
& s\left(\varphi_{h}, \psi_{h}\right) \lesssim\left\|\varphi_{h}\right\|_{\Phi}\left\|\psi_{h}\right\|_{\Phi}, \\
& s\left(\varphi_{h}, \varphi_{h}\right) \gtrsim\left\|\varphi_{h}\right\|_{\Phi}^{2} .
\end{aligned}
$$

\section{Preconditioning By Substructuring}

For both original and stabilized three fields formulation, we end up with an equation on the interface space $\Phi_{h}$ of the form

$$
S \underline{\varphi}_{h}=\underline{f}_{h}
$$

corresponding, through a relation of the form (23), to a bilinear form $s: \Phi_{h} \times \Phi_{h} \rightarrow$ $\mathbb{R}$ verifying for all $\varphi_{h}, \psi_{h} \in \Phi_{h}$ that

$$
\left|s\left(\varphi_{h}, \psi_{h}\right)\right| \lesssim\left\|\varphi_{h}\right\|_{\Phi}\left\|\psi_{h}\right\|_{\Phi}, \quad s\left(\varphi_{h}, \varphi_{h}\right) \gtrsim\left\|\varphi_{h}\right\|_{\Phi}^{2} .
$$

Our aim is to provide a preconditioner for the matrix $S$. The approach that we will follow is the one proposed, in the framework of conforming domain decomposition, 
by J.H. Bramble, J.E. Pasciak and A.H. Schatz in [9] and already studied in [1] for the mortar method case.

Let us consider a splitting of the interior part of the skeleton $\Sigma \backslash \Omega$ as the disjoint union of $M$ segments $e_{i}$ of extrema $a_{i}$ and $b_{i}$

$$
\Sigma \backslash \partial \Omega=\bigcup_{i=1}^{M} e_{i}
$$

(corresponding, for instance, to the splitting of $\Sigma$ as the union of segments of the form $\partial \Omega_{k} \cap \partial \Omega_{\ell}$ ). For each $k$ let $E_{k}$ individuate those segments that, together with $\partial \Omega \cap \partial \Omega_{k}$, build up the boundary of the subdomain $\Omega_{k}$ :

$$
\partial \Omega_{k} \backslash \partial \Omega=\bigcup_{i \in E_{k}} e_{i}, \quad \text { with } E_{k}=\left\{i:\left|e_{i} \cap \partial \Omega_{k}\right|>0\right\} .
$$

We make the following assumption:

(A4) There exists a constant $c_{4}$ such that it holds that

$$
\sup _{k} \sup _{i \in E_{k}} \frac{\left|\partial \Omega_{k}\right|}{\left|e_{i}\right|} \leq c_{4} \text {. }
$$

Note that (A4) implies that $\#\left(E_{k}\right) \leq c_{4}$ for all $k$. This will allow us several times to write inequalities of the form $\left\|\sum_{i \in E_{k}} q_{i}\right\|^{2} \lesssim \sum_{i \in E_{k}}\left\|q_{i}\right\|^{2}$, where $\|\cdot\|$ stands for various norms and seminorms (the constant in the inequality depending on $c_{4}$ ).

Following [9], the main idea for constructing a preconditioner for the matrix $S$ is to decompose $\Phi_{h}$ as the direct sum of a coarse space $\mathcal{L}_{H}$ and some local spaces $\Phi_{h, i}^{0}$ (one for each edge $e_{i}$ of the decomposition of $\Sigma$ ) and then considering the corresponding block-Jacobi type preconditioners. More precisely, let $\mathcal{L}_{H} \subset \Phi$,

$$
\mathcal{L}_{H}=\left\{\varphi \in C^{0}(\Sigma): \quad \forall i=1, \ldots, M,\left.\varphi\right|_{e_{i}} \in \mathbb{P}_{1}\left(e_{i}\right), \varphi=0 \text { on } \partial \Omega\right\},
$$

denote the subset of those functions of $\Phi$ whose restriction to each segment $e_{i}$ is linear. In order to construct the preconditioner we make the following assumptions on the approximation space $\Phi_{h}$ :

(A5) The following two conditions hold:

(a) $\mathcal{L}_{H} \subset \Phi_{h}$;

(b) for all $\varphi_{h} \in \Phi_{h}$ such that at the extrema $a_{i}$ and $b_{i}$ of some edge $e_{i}$ it holds that $\varphi_{h}\left(a_{i}\right)=\varphi_{h}\left(b_{i}\right)=0$, the function $\varphi_{h, i}^{0}$ defined as

$$
\varphi_{h, i}^{0}=\varphi_{h} \text { on } e_{i}, \quad \varphi_{h, i}^{0}=0 \text { on } \Sigma \backslash e_{i}
$$

verifies

$$
\varphi_{h, i}^{0} \in \Phi_{h} .
$$

(A6) $\Phi_{h} \subset H^{1}(\Sigma)$ and for all $\varphi_{h} \in \Phi_{h}$ the following two inverse inequalities hold for all $k, k=1, \ldots, K$, and for all $r, t, 0 \leq r<t \leq 1$ :

$$
\begin{aligned}
\left|\varphi_{h}\right|_{H^{t}\left(\partial \Omega_{k}\right)} & \lesssim h_{k}^{r-t}\left|\varphi_{h}\right|_{H^{r}\left(\partial \Omega_{k}\right)}, \quad \forall i \in E_{k} . \\
\left|\varphi_{h}\right|_{H^{t}\left(e_{i}\right)} & \lesssim h_{k}^{r-t}\left|\varphi_{h}\right|_{H^{r}\left(e_{i}\right)}, \quad \forall i \text {. }
\end{aligned}
$$

Remark 3.1. Assumption (A5) is not at all restrictive. In a finite element framework it reduces to asking that the extrema $a_{i}$ and $b_{i}$ of the segments $e_{i}$ into which the skeleton is split be nodes of the finite element grid for $\Phi_{h}$.

Remark 3.2. The parameter $h_{k}$ appearing in the inverse inequality (27) has here the meaning of the smallest mesh size of the restriction to $\partial \Omega_{k}$ of $\Phi_{h}$. 
Under assumption (A5) it is easy to see that $\Phi_{h}$ can be split as

$$
\Phi_{h}=\mathcal{L}_{H} \oplus \Phi_{h}^{0}, \quad \varphi_{h}=\varphi_{H}+\varphi_{h}^{0},
$$

with $\Phi_{h}^{0}$ defined as

$$
\Phi_{h}^{0}=\left\{\varphi_{h} \in \Phi_{h}, \varphi_{h}=0 \text { at the extrema } a_{i} \text { and } b_{i} \text { of each edge } e_{i} \text { of } \Sigma\right\} .
$$

The subspace $\Phi_{h}^{0}$ verifies

$$
\Phi_{h}^{0}=\left.\bigoplus_{i=1}^{M}\left\{\varphi_{h} \in \Phi_{h}^{0}: \varphi_{h}=0 \text { on } \Sigma \backslash e_{i}\right\} \sim \bigoplus_{i=1}^{M} \Phi_{h}^{0}\right|_{e_{i}},
$$

where the symbol $\sim$ means that the two spaces are isomorphic. Note that all $\varphi_{h} \in$ $\Phi_{h}^{0}$ verify $\left.\varphi_{h}\right|_{e_{i}} \in H_{00}^{1 / 2}\left(e_{i}\right)$ for all $i$, and in the following we will write $\left\|\varphi_{h}\right\|_{H_{00}^{1 / 2}\left(e_{i}\right)}$ for $\left\|\left.\varphi_{h}\right|_{e_{i}}\right\|_{H_{00}^{1 / 2}\left(e_{i}\right)}$ (and analogously for other norms defined on portions of $\Sigma$ ).

For each $i=1, \cdots, M$, we assume we are given a bilinear form $\check{s}_{i}:\left.\Phi_{h}^{0}\right|_{e_{i}} \times$ $\left.\Phi_{h}^{0}\right|_{e_{i}} \rightarrow \mathbb{R}$, satisfying the following continuity and positivity bounds uniformly in $h$ : for all $\eta,\left.\xi \in \Phi_{h}^{0}\right|_{e_{i}}$ :

$$
\check{s}_{i}(\xi, \eta) \lesssim\|\xi\|_{H_{00}^{1 / 2}\left(e_{i}\right)}\|\eta\|_{H_{00}^{1 / 2}\left(e_{i}\right)}, \quad \check{s}_{i}(\xi, \xi) \gtrsim\|\xi\|_{H_{00}^{1 / 2}\left(e_{i}\right)}^{2} .
$$

Moreover we assume that we are given a bilinear form $\check{s}_{H}: \mathcal{L}_{H} \times \mathcal{L}_{H} \rightarrow \mathbb{R}$ such that for all $\xi_{H}, \zeta_{H} \in \mathcal{L}_{H}$ it holds that

$$
\check{s}_{H}\left(\xi_{H}, \eta_{H}\right) \lesssim\left\|\xi_{H}\right\|_{\Phi}\left\|\zeta_{H}\right\|_{\Phi}, \quad \check{s}_{H}\left(\xi_{H}, \xi_{H}\right) \gtrsim\left\|\xi_{H}\right\|_{\Phi}^{2} .
$$

It is beyond the goals of this paper to fully discuss the different ways in which the bilinear forms $\check{s}_{i}$ and $\check{s}_{H}$ can be constructed. In the following section we will briefly recall a possible solution and we refer to [9, 10, 16] for further discussions on this topic.

Following [9], the preconditioner will be finally constructed with the aid of a bilinear form $\check{s}: \Phi_{h} \times \Phi_{h} \rightarrow \mathbb{R}$ assembled by blocks: for $\eta_{h}, \zeta_{h} \in \Phi_{h}$, with $\eta_{h}=$ $\eta_{H}+\eta_{h}^{0}, \zeta_{h}=\zeta_{H}+\zeta_{h}^{0}, \eta_{H}, \zeta_{H} \in \mathcal{L}_{H}$ and $\eta_{h}^{0}, \zeta_{h}^{0} \in \Phi_{h}^{0}$ we set

$$
\check{s}\left(\eta_{h}, \zeta_{h}\right)=\check{s}_{H}\left(\eta_{H}, \zeta_{H}\right)+\sum_{i=1}^{M} \check{s}_{i}\left(\left.\eta_{h}^{0}\right|_{e_{i}},\left.\zeta_{h}^{0}\right|_{e_{i}}\right) .
$$

The main result of this paper is the following theorem.

Theorem 3.1. For all $\varphi_{h} \in \Phi_{h}$ it holds that

$$
s\left(\varphi_{h}, \varphi_{h}\right) \lesssim \check{s}\left(\varphi_{h}, \varphi_{h}\right) \lesssim \max _{k}\left(1+\log \frac{H_{k}}{h_{k}}\right)^{2} s\left(\varphi_{h}, \varphi_{h}\right) .
$$

By a well-known argument, Theorem 3.1 implies that we can derive the following corollary, where we denote by $S$ and $\breve{S}$, respectively, the matrices corresponding to the Galerkin discretization of the bilinear forms $s$ and $\check{s}$.

Corollary 3.1. It holds that

$$
\operatorname{cond}\left(\check{S}^{-1} S\right) \lesssim \max _{k}\left(1+\log \frac{H_{k}}{h_{k}}\right)^{2}
$$


We recall that by the definition of the bilinear form $s$, the matrix $S$ is indeed the Schur complement matrix defined by either (14) or (21).

In order to prove Theorem 3.1 we will basically follow the guidelines of the proof of the analogous theorems of [9, 1]. The main difference is that here we consider a general discretization space, not necessarily consisting in piecewise linear functions. Moreover we will be working with functions and operators defined directly on the interface.

In view of the definition of $\check{s}_{i}$ and $\check{s}_{H}$ on the one hand and of property (26) of $s$ on the other hand, proving (30) is equivalent to proving that $\forall \varphi_{h} \in \Phi_{h}$ with $\varphi_{h}=\varphi_{H}+\varphi_{h}^{0}\left(\varphi_{H} \in \mathcal{L}_{H}\right.$ and $\varphi_{h}^{0}$ in $\left.\Phi_{h}^{0}\right)$, it holds that

$$
\left\|\varphi_{h}\right\|_{\Phi}^{2} \lesssim\left\|\varphi_{H}\right\|_{\Phi}^{2}+\sum_{i=1}^{M}\left\|\varphi_{h}^{0}\right\|_{H_{00}^{1 / 2}\left(e_{i}\right)}^{2} \lesssim \max _{k}\left(1+\log \frac{H_{k}}{h_{k}}\right)^{2}\left\|\varphi_{h}\right\|_{\Phi}^{2} .
$$

We start by observing that it holds that

$$
\left\|\varphi_{h}\right\|_{\Phi}^{2} \lesssim\left\|\varphi_{H}\right\|_{\Phi}^{2}+\left\|\varphi_{h}^{0}\right\|_{\Phi}^{2} \lesssim\left\|\varphi_{H}\right\|_{\Phi}^{2}+\sum_{i=1}^{M}\left\|\varphi_{h}^{0}\right\|_{H_{00}^{1 / 2}\left(e_{i}\right)}^{2},
$$

where the last bound descends by splitting $\varphi_{h}^{0} \in \Phi_{h}^{0}$ as $\varphi_{h}^{0}=\sum_{i=1}^{M} \varphi_{h, i}^{0}$ which implies (since $\#\left(E_{k}\right) \leq c_{4}$ and since each $i$ belongs to $E_{k}$ for at most two $k$ 's)

$$
\left\|\varphi_{h}^{0}\right\|_{\Phi}^{2} \lesssim \sum_{k}\left|\varphi_{h}^{0}\right|_{H^{1 / 2}\left(\partial \Omega_{k}\right)}^{2} \lesssim \sum_{k} \sum_{i \in E_{k}}\left|\varphi_{h, i}^{0}\right|_{H^{1 / 2}\left(\partial \Omega_{k}\right)}^{2} \lesssim \sum_{i=1}^{M}\left\|\varphi_{h}^{0}\right\|_{H_{00}^{1 / 2}\left(e_{i}\right)}^{2},
$$

the last inequality deriving from the $H_{00}^{1 / 2}\left(e_{i}\right)$ to $H^{1 / 2}\left(\partial \Omega_{k}\right)$ boundedness of the trivial extension-by-zero operator.

Moreover, by direct computation, using the linearity of $\varphi_{H}$, one can see that it holds that

$$
\left\|\varphi_{H}\right\|_{\Phi}^{2} \lesssim \sum_{k=1}^{K}\left|\varphi_{H}\right|_{H^{1 / 2}\left(\partial \Omega_{k}\right)}^{2} \lesssim \sum_{i=1}^{M}\left(\varphi_{H}\left(a_{i}\right)-\varphi_{H}\left(b_{i}\right)\right)^{2}
$$

$a_{i}$ and $b_{i}$ being the extrema of $e_{i}$. Then, proving Theorem 3.1 reduces to proving the following result:

Theorem 3.2. For all $\varphi_{h} \in \Phi_{h}$ with $\varphi_{h}=\varphi_{H}+\varphi_{h}^{0}\left(\varphi_{H} \in \mathcal{L}_{H}\right.$ and $\left.\varphi_{h}^{0} \in \Phi_{h}^{0}\right)$, it holds that

$$
\sum_{i=1}^{M}\left(\varphi_{H}\left(a_{i}\right)-\varphi_{H}\left(b_{i}\right)\right)^{2}+\sum_{i=1}^{M}\left\|\varphi_{h}^{0}\right\|_{H_{00}^{1 / 2}\left(e_{i}\right)}^{2} \lesssim \max _{k}\left(1+\log \frac{H_{k}}{h_{k}}\right)^{2}\left\|\varphi_{h}\right\|_{\Phi}^{2} .
$$

In order to prove Theorem 3.2, we will need several bounds. We start by proving the following Lemma.

Lemma 3.1. Assume that $\Phi_{h}$ satisfies assumptions (A5) and (A6). Then the following bounds hold:

(i) for all $\varphi_{h} \in \Phi_{h}$ such that $\varphi_{h}(p)=0$ for some $p \in \bar{e}_{i}$ with $i \in E_{k}$, we have

$$
\left\|\varphi_{h}\right\|_{L^{\infty}\left(e_{i}\right)}^{2} \lesssim\left(1+\log \frac{H_{k}}{h_{k}}\right)\left|\varphi_{h}\right|_{H^{1 / 2}\left(e_{i}\right)}^{2} ;
$$


(ii) for all $\varphi_{h} \in \Phi_{h}$ it holds that

$$
\left(\varphi_{h}\left(a_{i}\right)-\varphi_{h}\left(b_{i}\right)\right)^{2} \lesssim\left(1+\log \frac{H_{k}}{h_{k}}\right)\left|\varphi_{h}\right|_{H^{1 / 2}\left(e_{i}\right)}^{2},
$$

$a_{i}$ and $b_{i}$ being the extrema of $e_{i}, i \in E_{k}$;

(iii) for all $\varphi_{h}^{0} \in \Phi_{h}^{0}$ it holds that

$$
\sum_{i \in E_{k}}\left\|\varphi_{h}^{0}\right\|_{H_{00}^{1 / 2}\left(e_{i}\right)}^{2} \lesssim\left(1+\log \frac{H_{k}}{h_{k}}\right)^{2}\left|\varphi_{h}^{0}\right|_{H^{1 / 2}\left(\partial \Omega_{k}\right)}^{2} .
$$

Proof. Let $i \in E_{k}$. By assumptions (A1)(a) and (A4) we have that $\left|e_{i}\right| \simeq H_{k}$. We start by observing that for any $\zeta \in H^{1 / 2+\varepsilon}\left(e_{i}\right)$ it holds that

$$
\|\zeta\|_{L^{\infty}\left(e_{i}\right)}^{2} \lesssim H_{k}^{-1}\|\zeta\|_{L^{2}\left(e_{i}\right)}^{2}+\frac{H_{k}^{2 \varepsilon}}{\varepsilon}|\zeta|_{H^{1 / 2+\varepsilon}\left(e_{i}\right)}^{2} .
$$

This is easily seen by observing that on the reference segment $\hat{e}=] 0,1[$ the continuity bound of the injection $H^{1 / 2+\varepsilon}(\hat{e}) \subset L^{\infty}(\hat{e})$ depends on $\varepsilon$ as follows (see Appendix A, LemmaA.1): for all $\zeta \in H^{1 / 2+\varepsilon}(\hat{e})$

$$
\|\zeta\|_{L^{\infty}(\hat{e})}^{2} \lesssim\|\zeta\|_{L^{2}(\hat{e})}^{2}+\frac{1}{\varepsilon}|\zeta|_{H^{1 / 2+\varepsilon}(\hat{e})}^{2} .
$$

A change of variable then yields (37). Let now $\varphi_{h} \in \Phi_{h}$ and $\alpha \in \mathbb{R}$. By assumption (A6) it holds that

$$
\frac{H_{k}^{2 \varepsilon}}{\varepsilon}\left|\varphi_{h}-\alpha\right|_{H^{1 / 2+\varepsilon}\left(e_{i}\right)}^{2}=\frac{H_{k}^{2 \varepsilon}}{\varepsilon}\left|\varphi_{h}\right|_{H^{1 / 2+\varepsilon}\left(e_{i}\right)}^{2} \lesssim \frac{H_{k}^{2 \varepsilon} h_{k}^{-2 \varepsilon}}{\varepsilon}\left|\varphi_{h}\right|_{H^{1 / 2}\left(e_{i}\right)}^{2} .
$$

After choosing $\varepsilon=1 / \log \left(H_{k} / h_{k}\right)$, inequality (37) for $\zeta=\varphi_{h}-\alpha$ combined with (38) yields:

$$
\left\|\varphi_{h}-\alpha\right\|_{L^{\infty}\left(e_{i}\right)}^{2} \lesssim H_{k}^{-1}\left\|\varphi_{h}-\alpha\right\|_{L^{2}\left(e_{i}\right)}^{2}+\log \frac{H_{k}}{h_{k}}\left|\varphi_{h}\right|_{H^{1 / 2}\left(e_{i}\right)}^{2} .
$$

In view of (39), (34) can then be proven by applying exactly the arguments of [9], which we repeat here for completeness: let us choose $\alpha$ to be the average of $\varphi_{h}$ on $e_{i}$. Applying Poincaré inequality gives

$$
H_{k}^{-1 / 2}\left\|\varphi_{h}-\alpha\right\|_{L^{2}\left(e_{i}\right)} \lesssim\left|\varphi_{h}\right|_{H^{1 / 2}\left(e_{i}\right)}
$$

which yields

$$
\left\|\varphi_{h}-\alpha\right\|_{L^{\infty}\left(e_{i}\right)}^{2} \lesssim\left(1+\log \frac{H_{k}}{h_{k}}\right)\left|\varphi_{h}\right|_{H^{1 / 2}\left(e_{i}\right)}^{2} .
$$

Now we remark that $\varphi_{h}(p)=0$ for some $p \in \bar{e}_{i}$ implies

$$
|\alpha| \lesssim\left\|\varphi_{h}-\alpha\right\|_{L^{\infty}\left(e_{i}\right)}
$$

and we get (34) by triangular inequality.

(ii) then follows by applying (i) to the function $\varphi(x)-\varphi\left(a_{i}\right)$ (which vanishes in $\left.p=a_{i}\right)$.

As far as (iii) is concerned, we start by remarking that for $\varphi_{h}^{0} \in \Phi_{h}^{0}$ and for $i \in E_{k}$, denoting by $\varphi_{h, i}^{0}$ the function coinciding with $\varphi_{h}^{0}$ on $e_{i}$ and identically vanishing on $\partial \Omega_{k} \backslash e_{i}$ and using (3) and the inverse inequality (27), we obtain the following bounds

$$
\left\|\varphi_{h}^{0}\right\|_{H_{00}^{1 / 2}\left(e_{i}\right)} \lesssim\left|\varphi_{h, i}^{0}\right|_{H^{1 / 2}\left(\partial \Omega_{k}\right)} \lesssim h_{k}^{-\varepsilon}\left|\varphi_{h, i}^{0}\right|_{H^{1 / 2-\varepsilon}\left(\partial \Omega_{k}\right)} \lesssim\left|\varphi_{h}^{0}\right|_{H_{0}^{1 / 2-\varepsilon}\left(e_{i}\right)},
$$


where the second inequality descends from (27) by applying a standard functional spaces interpolation argument, since the spaces $H_{0}^{r}\left(e_{i}\right), 0<r<1 / 2$, can be obtained as the interpolants of order $2 r$ between $L^{2}\left(e_{i}\right)$ and $H_{00}^{1 / 2}\left(e_{i}\right)$. Again we bound the $H_{0}^{1 / 2-\varepsilon}\left(e_{i}\right)$ seminorm of $\varphi_{h}^{0}$ by a change of variable, using the corresponding bound on the reference segment $\hat{e}=] 0,1\left[\right.$. We recall once more that $H_{0}^{1 / 2-\varepsilon}(\hat{e})$ and $H^{1 / 2-\varepsilon}(\hat{e})$ coincide, with equivalent norms, the constant in the equivalence depending on $\varepsilon$. In particular it is possible to prove (see Appendix A, Corollary A.1) that the following bound holds for all $\zeta \in H^{1 / 2}(\hat{e})$ and for all $\alpha \in \mathbb{R}$ :

$$
|\zeta|_{H_{0}^{1 / 2-\varepsilon}(\hat{e})} \lesssim \frac{1}{\varepsilon}\|\zeta-\alpha\|_{H^{1 / 2}(\hat{e})}+\frac{1}{\sqrt{\varepsilon}}|\alpha|,
$$

which rescales as

$$
|\zeta|_{H_{0}^{1 / 2-\varepsilon}\left(e_{i}\right)} \lesssim \frac{H_{k}^{\varepsilon}}{\varepsilon}\left(H_{k}^{-1 / 2}\|\zeta-\alpha\|_{L^{2}\left(e_{i}\right)}+|\zeta-\alpha|_{H^{1 / 2}\left(e_{i}\right)}\right)+\frac{H_{k}^{\varepsilon}}{\sqrt{\varepsilon}}|\alpha| .
$$

Again, taking $\zeta=\varphi_{h}^{0}$ and $\alpha$ as its average on $e_{i}$, we can apply on the one hand Poincaré inequality (40) and on the other hand the bound (42), which holds since $\varphi_{h}^{0}\left(a_{i}\right)=0$, and we get

$$
\left\|\varphi_{h}^{0}\right\|_{H_{00}^{1 / 2}\left(e_{i}\right)} \lesssim \frac{H^{\varepsilon} h_{k}^{-\varepsilon}}{\varepsilon}\left|\varphi_{h}^{0}\right|_{H^{1 / 2}\left(e_{i}\right)}+\frac{H^{\varepsilon} h_{k}^{-\varepsilon}}{\sqrt{\varepsilon}}\left\|\varphi_{h}^{0}-\alpha\right\|_{L^{\infty}\left(e_{i}\right)} .
$$

We then take once again $\varepsilon=1 / \log \left(H_{k} / h_{k}\right)$, and, thanks to bound (41), we obtain

$$
\left\|\varphi_{h}^{0}\right\|_{H_{00}^{1 / 2}\left(e_{i}\right)} \lesssim\left(1+\log \frac{H_{k}}{h_{k}}\right)\left|\varphi_{h}^{0}\right|_{H^{1 / 2}\left(e_{i}\right)} .
$$

Since $\sum_{i \in E_{k}}|\cdot|_{H^{1 / 2}\left(e_{i}\right)}^{2} \lesssim|\cdot|_{H^{1 / 2}\left(\partial \Omega_{k}\right)}^{2}$, by squaring and then taking the sum over $i \in E_{k}$, we obtain (36).

Remark 3.3. Since for $s<1 / 2$ the two spaces $H^{s}\left(e_{i}\right)$ and $H_{0}^{s}\left(e_{i}\right)$ coincide, the seminorm $|\cdot|_{H_{0}^{s}\left(e_{i}\right)}$ in equation (43) could be replaced by the standard $H^{s}\left(e_{i}\right)$ seminorm. In that case, however, the implicit constant in the bound would depend on $\varepsilon$.

Analogously to what happens in [9, the main ingredient of the proof of Theorem 3.2 is then the following lemma.

Lemma 3.2. Let $\varphi_{h}^{0} \in \Phi_{h}^{0}$ and $\zeta_{H} \in \mathcal{L}_{H}$. Then for all $k$ it holds that

$$
\sum_{i \in E_{k}}\left\|\varphi_{h}^{0}\right\|_{H_{00}^{1 / 2}\left(e_{i}\right)}^{2} \lesssim\left(1+\log \frac{H_{k}}{h_{k}}\right)^{2}\left|\varphi_{h}^{0}+\zeta_{H}\right|_{H^{1 / 2}\left(\partial \Omega_{k}\right)}^{2} .
$$

Provided such lemma holds, (33) is obtained, thanks to (ii) of Lemma 3.1. by applying (45) for $\zeta_{H}=\varphi_{H}$. In fact, for $\varphi_{h}=\varphi_{H}+\varphi_{h}^{0}, \varphi_{H} \in \mathcal{L}_{H}, \varphi_{h}^{0} \in \Phi_{h}^{0}$, we can write

$$
\begin{aligned}
\sum_{i=1}^{M}\left\|\varphi_{h}^{0}\right\|_{H_{00}^{1 / 2}\left(e_{i}\right)}^{2} & =\frac{1}{2} \sum_{k=1}^{K} \sum_{i \in E_{k}}\left\|\varphi_{h}^{0}\right\|_{H_{00}^{1 / 2}\left(e_{i}\right)}^{2} \\
& \lesssim \sum_{k}\left(1+\log \frac{H_{k}}{h_{k}}\right)^{2}\left|\varphi_{h}\right|_{H^{1 / 2}\left(\partial \Omega_{k}\right)}^{2} \\
& \lesssim \max _{k}\left(1+\log \frac{H_{k}}{h_{k}}\right)^{2}\left\|\varphi_{h}\right\|_{\Phi}^{2},
\end{aligned}
$$


which, combined with (35), yields (33). The only thing that we need to do then is to prove Lemma 3.2 .

Proof of Lemma 3.2. Let $k, 1 \leq k \leq K$. We start by introducing a function $\zeta^{0} \in$ $\Phi_{h}^{k, 0}$,

$$
\Phi_{h}^{k, 0}=\left\{\left.\varphi_{h} \in \Phi_{h}\right|_{\partial \Omega_{k}}: \varphi_{h}=0 \text { at the cross points of } \partial \Omega_{k}\right\}=\left.\Phi_{h}^{0}\right|_{\partial} \Omega_{k},
$$

$\zeta^{0}$ depending on $\zeta_{H}$, defined as the unique element of $\Phi_{h}^{k, 0}$ satisfying

$$
\left(\zeta^{0}, \xi\right)_{H^{1 / 2}\left(\partial \Omega_{k}\right)}=\left(\zeta_{H}, \xi\right)_{H^{1 / 2}\left(\partial \Omega_{k}\right)}, \quad \forall \xi \in \Phi_{h}^{k, 0},
$$

where $(\cdot, \cdot)_{H^{1 / 2}\left(\partial \Omega_{k}\right)}$ is the bilinear form

$$
(\zeta, \xi)_{H^{1 / 2}\left(\partial \Omega_{k}\right)}=\int_{\partial \Omega_{k}} \int_{\partial \Omega_{k}} \frac{(\zeta(x)-\zeta(y))(\xi(x)-\xi(y))}{|x-y|^{2}} d x d y
$$

inducing the $H^{1 / 2}\left(\partial \Omega_{k}\right)$ seminorm. It is easy to check that $|\cdot|_{H^{1 / 2}\left(\partial \Omega_{k}\right)}$ is a norm on $\Phi_{h}^{k, 0}$ and then we can conclude by standard arguments that

$$
\left|\zeta^{0}\right|_{H^{1 / 2}\left(\partial \Omega_{k}\right)} \lesssim\left|\zeta_{H}\right|_{H^{1 / 2}\left(\partial \Omega_{k}\right)}
$$

Now, adding and subtracting $\zeta^{0}$ at the left-hand side of (45), we can write:

$$
\sum_{i \in E_{k}}\left\|\varphi_{h}^{0}\right\|_{H_{00}^{1 / 2}\left(e_{i}\right)}^{2} \lesssim \sum_{i \in E_{k}}\left\|\varphi_{h}^{0}+\zeta^{0}\right\|_{H_{00}^{1 / 2}\left(e_{i}\right)}^{2}+\sum_{i \in E_{k}}\left\|\zeta^{0}\right\|_{H_{00}^{1 / 2}\left(e_{i}\right)}^{2}
$$

Since $\varphi_{h}^{0}+\zeta^{0} \in \Phi_{h}^{k, 0}$, by the definition of $\zeta^{0}$ we have that

$$
\left(\zeta_{H}-\zeta^{0}, \varphi_{h}^{0}+\zeta^{0}\right)_{H^{1 / 2}\left(\partial \Omega_{k}\right)}=0
$$

which yields

$$
\left|\varphi_{h}^{0}+\zeta_{H}\right|_{H^{1 / 2}\left(\partial \Omega_{k}\right)}^{2} \geq\left|\varphi_{h}^{0}+\zeta^{0}\right|_{H^{1 / 2}\left(\partial \Omega_{k}\right)}^{2} .
$$

The first sum on the right-hand side of (48) can then be bound thanks to Lemma 3.1(iii); we have

$$
\begin{aligned}
\sum_{i \in E_{k}}\left\|\varphi_{h}^{0}+\zeta^{0}\right\|_{H_{00}^{1 / 2}\left(e_{i}\right)}^{2} & \lesssim\left(1+\log \frac{H_{k}}{h_{k}}\right)^{2}\left|\varphi_{h}^{0}+\zeta^{0}\right|_{H^{1 / 2}\left(\partial \Omega_{k}\right)}^{2} \\
& \lesssim\left(1+\log \frac{H_{k}}{h_{k}}\right)^{2}\left|\varphi_{h}^{0}+\zeta_{H}\right|_{H^{1 / 2}\left(\partial \Omega_{k}\right)}^{2}
\end{aligned}
$$

Let us bound the second sum on the right-hand side of (48). We recall that we have

$$
\left\|\zeta^{0}\right\|_{H_{00}^{1 / 2}\left(e_{i}\right)}^{2}=\left|\zeta^{0}\right|_{H^{1 / 2}\left(e_{i}\right)}^{2}+I_{1}\left(\zeta^{0}\right)+I_{2}\left(\zeta^{0}\right)
$$

with

$$
I_{1}\left(\zeta^{0}\right)=\int_{a_{i}}^{b_{i}} \frac{\left|\zeta^{0}(x)\right|^{2}}{\left|x-a_{i}\right|} d x, \quad I_{2}\left(\zeta^{0}\right)=\int_{a_{i}}^{b_{i}} \frac{\left|\zeta^{0}(x)\right|^{2}}{\left|x-b_{i}\right|} d x
$$


(we recall that $a_{i}$ and $b_{i}$ denote the two vertices of the edge $e_{i}$ ). Now, using equations (47), (32) and (35), since $\varphi_{h}^{0}$ vanishes at $a_{i}$ and $b_{i}$, we can write

$$
\begin{aligned}
\sum_{i \in E_{k}}\left|\zeta^{0}\right|_{H^{1 / 2}\left(e_{i}\right)}^{2} & \lesssim\left|\zeta^{0}\right|_{H^{1 / 2}\left(\partial \Omega_{k}\right)}^{2} \lesssim\left|\zeta_{H}\right|_{H^{1 / 2}\left(\partial \Omega_{k}\right)}^{2} \\
& \lesssim \sum_{i \in E_{k}}\left(\zeta_{H}\left(a_{i}\right)-\zeta_{H}\left(b_{i}\right)\right)^{2} \\
& =\sum_{i \in E_{k}}\left(\left[\varphi_{h}^{0}+\zeta_{H}\right]\left(a_{i}\right)-\left[\varphi_{h}^{0}+\zeta_{H}\right]\left(b_{i}\right)\right)^{2} \\
& \lesssim\left(1+\log \frac{H_{k}}{h_{k}}\right)\left|\varphi_{h}^{0}+\zeta_{H}\right|_{H^{1 / 2}\left(\partial \Omega_{k}\right)}^{2} .
\end{aligned}
$$

Let us now bound $I_{1}\left(\zeta^{0}\right)$. For notational simplicity let us identify $\left.e_{i}=\right] 0, T[$ with $a_{i}=0$ and $b_{i}=T$. We have

$$
\begin{aligned}
I_{1}\left(\zeta^{0}\right)= & \int_{0}^{T} \frac{\left|\zeta^{0}(x)\right|^{2}}{|x|} d x \\
= & \int_{0}^{T} \frac{\left|\zeta^{0}(x)+\zeta_{H}(x)-\zeta_{H}(x)+\zeta_{H}(0)-\zeta_{H}(0)\right|^{2}}{|x|} d x \\
\lesssim & \int_{0}^{T} \frac{\left|\zeta^{0}(x)-\zeta_{H}(x)+\zeta_{H}(0)\right|^{2}}{|x|} d x \\
& \quad+\int_{0}^{T} \frac{\left|\zeta_{H}(x)-\zeta_{H}(0)\right|^{2}}{|x|} d x .
\end{aligned}
$$

Let us set $\zeta^{\perp}=\zeta^{0}-\zeta_{H}$. Remarking that $\zeta^{\perp}(0)=-\zeta_{H}(0)$, we have

$$
\begin{aligned}
\int_{0}^{T} \frac{\left|\zeta^{0}(x)-\zeta_{H}(x)+\zeta_{H}(0)\right|^{2}}{|x|} d x= & \int_{0}^{T} \frac{\left|\zeta^{\perp}(x)-\zeta^{\perp}(0)\right|^{2}}{|x|} d x \\
= & \int_{0}^{h_{k}} \frac{\left|\zeta^{\perp}(x)-\zeta^{\perp}(0)\right|^{2}}{|x|} d x \\
& +\int_{h_{k}}^{T} \frac{\left|\zeta^{\perp}(x)-\zeta^{\perp}(0)\right|^{2}}{|x|} d x .
\end{aligned}
$$

The first term is bound, using Cauchy-Schwarz's inequality and inequality (28), by

$$
\begin{aligned}
\int_{0}^{h_{k}} \frac{\left|\zeta^{\perp}(x)-\zeta^{\perp}(0)\right|^{2}}{|x|} d x & =\int_{0}^{h_{k}} \frac{1}{|x|}\left|\int_{0}^{x} \zeta_{x}^{\perp}(\tau) d \tau\right|^{2} d x \\
& \lesssim \int_{0}^{h_{k}} \int_{0}^{x}\left|\zeta_{x}^{\perp}(\tau)\right|^{2} d \tau d x \\
& \lesssim h_{k}\left|\zeta^{\perp}\right|_{H^{1}\left(e_{i}\right)}^{2} \lesssim\left|\zeta^{\perp}\right|_{H^{1 / 2}\left(e_{i}\right)}^{2},
\end{aligned}
$$

while, using (34), we bound the second by

$$
\begin{aligned}
\int_{h_{k}}^{T} \frac{\left|\zeta^{\perp}(x)-\zeta^{\perp}(0)\right|^{2}}{|x|} d x & \lesssim\left\|\zeta^{\perp}-\zeta^{\perp}(0)\right\|_{L^{\infty}\left(e_{i}\right)}^{2} \int_{h_{k}}^{T} \frac{1}{x} d x \\
& \lesssim\left(1+\log \frac{H_{k}}{h_{k}}\right)^{2}\left|\zeta^{\perp}\right|_{H^{1 / 2}\left(e_{i}\right)}^{2}
\end{aligned}
$$


where we used $T \simeq H_{k}$. The second integral in (60) can be bound directly using the linearity of $\zeta_{H}$.

$$
\int_{0}^{T} \frac{\left|\zeta_{H}(x)-\zeta_{H}(0)\right|^{2}}{|x|} d x \lesssim\left(\zeta_{H}\left(b_{i}\right)-\zeta_{H}\left(a_{i}\right)\right)^{2} \lesssim\left(1+\log \frac{H_{k}}{h_{k}}\right)\left|\zeta_{H}+\varphi_{h}^{0}\right|_{H^{1 / 2}\left(e_{i}\right)}^{2}
$$

where again we used (35) and the fact that $\varphi_{h}^{0}$ vanishes at $a_{i}$ and $b_{i}$. Then, adding up the different contributions, we conclude that

$$
I_{1}\left(\zeta^{0}\right) \lesssim\left(1+\log \frac{H_{k}}{h_{k}}\right)^{2}\left|\zeta^{0}-\zeta_{H}\right|_{H^{1 / 2}\left(e_{i}\right)}^{2}+\left(1+\log \frac{H_{k}}{h_{k}}\right)\left|\zeta_{H}+\varphi_{h}^{0}\right|_{H^{1 / 2}\left(e_{i}\right)}^{2} .
$$

By using the same arguments we get a similar bound for $I_{2}$ :

$$
I_{2}\left(\zeta^{0}\right) \lesssim\left(1+\log \frac{H_{k}}{h_{k}}\right)^{2}\left|\zeta^{0}-\zeta_{H}\right|_{H^{1 / 2}\left(e_{i}\right)}^{2}+\left(1+\log \frac{H_{k}}{h_{k}}\right)\left|\zeta_{H}+\varphi_{h}^{0}\right|_{H^{1 / 2}\left(e_{i}\right)}^{2} .
$$

We can now insert bounds (54), (62) and (63) back into (52) and sum over $i \in$ $E_{k}$. By observing that for any $w \in H^{1 / 2}\left(\partial \Omega_{k}\right)$ it holds that $\sum_{i \in E_{k}}|w|_{H^{1 / 2}\left(e_{i}\right)}^{2} \lesssim$ $|w|_{H^{1 / 2}\left(\partial \Omega_{k}\right)}^{2}$, we obtain the bound

$$
\begin{aligned}
\sum_{i \in E_{k}}\left\|\zeta^{0}\right\|_{H_{00}^{1 / 2}\left(e_{i}\right)}^{2} \lesssim & \left(1+\log \frac{H_{k}}{h_{k}}\right)\left|\varphi_{h}^{0}+\zeta_{H}\right|_{H^{1 / 2}\left(\partial \Omega_{k}\right)}^{2} \\
& +\left(1+\log \frac{H_{k}}{h_{k}}\right)^{2}\left|\zeta^{0}-\zeta_{H}\right|_{H^{1 / 2}\left(\partial \Omega_{k}\right)}^{2}
\end{aligned}
$$

We now observe once again that equation (49) implies that $\left|\zeta_{H}-\zeta^{0}\right|_{H^{1 / 2}\left(\partial \Omega_{k}\right)} \lesssim$ $\left|\zeta_{H}+\varphi_{h}^{0}\right|_{H^{1 / 2}\left(\partial \Omega_{k}\right)}^{2}$ and we get the thesis.

\section{Numerical Tests}

We will test the preconditioner we propose on the following problem: find $u$ such that

$$
\left\{\begin{aligned}
-\Delta u=f, & \text { in } \Omega=] 0,1[\times] 0,1[, \\
u=0, & \text { on } \Gamma=\partial \Omega .
\end{aligned}\right.
$$

We consider a uniform, geometrically conforming, decomposition of $\Omega=] 0,1[\times$ ]0,1 [ in $K=N \times N$ equal square subdomains of size $H \times H, H=1 / N$.

In each subdomain $\Omega_{k}$ we take a uniform mesh $\mathcal{T}^{k}$ composed of $n_{k} \times n_{k}$ equal square elements of size $\delta_{k} \times \delta_{k}, \delta_{k}=H / n_{k}=1 /\left(N n_{k}\right)$. We then define $V_{h}^{k}$ to be the space of $\mathbb{Q}_{1}$ finite elements on the mesh $\mathcal{T}^{k}$,

$$
V_{h}^{k}=\left\{u_{h} \in C^{0}(\Omega):\left.u_{h}\right|_{\tau} \in \mathbb{Q}_{1}(\tau), \forall \tau \in \mathcal{T}^{k}\right\},
$$

where $\mathbb{Q}_{1}(\tau)$ denotes the space of polynomials of degree less than or equal to one in each variable. The multiplier space $\Lambda_{h}^{k}$ is then defined as the trace on $\partial \Omega_{k}$ of $V_{h}^{k}$. With such a choice it is possible to prove that (A3) holds (see 4]).

The skeleton $\Sigma \backslash \partial \Omega$ is split as $\Sigma \backslash \partial \Omega=\bigcup_{i=1}^{2(N-1) N} e_{i}$ with $e_{i}=\Gamma_{k} \cap \Gamma_{\ell}$ for some $k=k(i)$ and $\ell=\ell(i)$. We remark that $\left|e_{i}\right|=H$ for all $i$. We consider a grid $\mathcal{G}$ on $\Sigma$ obtained by uniformly splitting each $e_{i}$ into $L_{i}$ elements of size $h_{i}$ and we define $\Phi_{h}$ to be the space of $\mathbb{P}_{1}$ finite elements on such a grid:

$$
\Phi_{h}=\left\{\varphi_{h} \in C^{0}(\Sigma):\left.\varphi_{h}\right|_{\kappa} \in \mathbb{P}_{1}(\kappa), \forall \kappa \in \mathcal{G},\left.\varphi_{h}\right|_{\partial \Omega}=0\right\} .
$$

With this choice, assumption (A6) holds for $h_{k}=\min _{i \in E_{k}} h_{i}$. 
Letting $\underline{\varphi}_{h, i}^{0}\left(\operatorname{resp} . \underline{\psi}_{h, i}^{0}\right)$ denote the vector of the values of $\varphi_{h}^{0}\left(\operatorname{resp} \psi_{h}^{0}\right) \in \Phi_{h}^{0}$ at the interior nodes of the grid $\left.\mathcal{G}\right|_{e_{i}}$, we define the bilinear form $\check{s}_{i}:\left.\Phi_{h, i}^{0}\right|_{e_{i}} \times\left.\Phi_{h, i}^{0}\right|_{e_{i}}$ as

$$
\check{s}_{i}\left(\left.\varphi_{h}^{0}\right|_{e_{i}},\left.\psi_{h}^{0}\right|_{e_{i}}\right)=\left(\underline{\psi}_{h, i}^{0}\right)^{T}\left(R_{i}\right)^{1 / 2} \underline{\varphi}_{h, i}^{0},
$$

where $R_{i}$ is the stiffness matrix associated to the discretization of the operator $-d^{2} / d x^{2}$ by $\mathbb{P}_{1}$ finite element on $e_{i}$ with homogeneous Dirichlet boundary conditions at the extrema $a_{i}$ and $b_{i}$. With $R_{i}$ being positive definite, its square root is well defined and it is computed and stored at the start.

As far as the coarse preconditioner $\check{s}_{H}$ is concerned, we considered two choices:

- (BPS) Following [9], we set

$$
\check{s}_{H}\left(\eta_{H}, \zeta_{H}\right):=2 \sum_{i=1}^{M}\left(\eta_{H}\left(a_{i}\right)-\eta_{H}\left(b_{i}\right)\right)\left(\zeta_{H}\left(a_{i}\right)-\zeta_{H}\left(b_{i}\right)\right) .
$$

- (Laplace) Denoting by $\tilde{\eta}_{H}$ and $\tilde{\zeta}_{H}$ the $H_{0}^{1}(\Omega)$ functions obtained by lifting $\eta_{H}$ and $\zeta_{H}$ harmonically in each subdomain, we set

$$
\check{s}_{H}\left(\eta_{H}, \zeta_{H}\right):=\int_{\Omega} \nabla \tilde{\eta}_{H} \nabla \tilde{\zeta}_{H} d \mathbf{x}
$$

note that the set $\left\{\tilde{\eta}_{H}, \eta_{H} \in \mathcal{L}_{H}\right\}$ coincides with the set of $\mathbb{Q}_{1}$ finite elements on the coarse grid corresponding to the decomposition $\Omega=\bigcup \Omega_{k}$, and then applying the coarse preconditioner reduces to numerically solving the Laplace equation on such space.

For all tests we set $f=1$. We solved the linear system by a preconditioned conjugate gradient method, using $\varphi_{h}=0$ as initial guess. We report the number of iterations needed to reduce the residual of a factor $10^{-5}$.

\subsection{Plain formulation.}

4.1.1. Conforming decomposition. We start by considering the case of a conforming domain decomposition, that is, in the framework described above we set $n_{k}=n$ for all $k$ and $L_{i}=n$ for all $i$. In this case $\delta_{k}=h_{i}=h=H / n$. With such a choice, it is not difficult to check that the inf-sup condition (12) is satisfied uniformly in $h$ and then we are in the range of Lemma 2.1. It is easy to realize that the discrete solution verifies $u_{h}^{k}=\varphi_{h}$ on $\partial \Omega_{k}$, and then the function $u_{h}$, defined by $\left.u_{h}\right|_{\Omega_{k}}=u_{h}^{k}$, verifies $u_{h} \in H_{0}^{1}(\Omega)$. In such a case we obtain the same solution that we would get using a conforming finite element method on a regular grid of $N n \times N n$ square elements of dimension $h \times h$. The use of a nonconforming method is therefore, in this case, only a device to implement a solver for the discrete problem in an easily parallelizable way.

In order to study both the dependence on $H$ (size of the subdomains) and on $h$ we tested the preconditioner for different values of $n$ in the range $[5,40]$ and $N$ in the range $[4,32]$. We tested both coarse preconditioners for all combinations of $N$ and $n$. The results are summarized in Tables 1 and 2, As one can see, they are in close agreement with the theory (note that in this case $\max _{k} h_{k} / H_{k}=h / H=n$ ). 
TABLE 1. Number of conjugate gradient iterations needed for reducing the residual of a factor $10^{-5}$, with coarse preconditioner given by (68) for different combinations of the number $K=N^{2}$ of subdomains and $n$ of elements per edge ( $n^{2}$ elements per subdomain).

\begin{tabular}{c|cccc} 
& $n=5$ & $n=10$ & $n=20$ & $n=40$ \\
$K=N^{2}$ & \# Iter. & \# Iter. & \# Iter. & \# Iter. \\
\hline 16 & 10 & 12 & 14 & 15 \\
64 & 14 & 17 & 20 & 23 \\
144 & 15 & 18 & 22 & 26 \\
256 & 15 & 19 & 23 & 27 \\
400 & 16 & 20 & 23 & 27 \\
576 & 16 & 20 & 24 & 27 \\
784 & 16 & 20 & 24 & 27 \\
1024 & 16 & 20 & 24 & 27 \\
\hline
\end{tabular}

TABLE 2. Number of conjugate gradient iterations needed for reducing the residual of a factor $10^{-5}$, with coarse preconditioner given by (67) for different combinations of the number $K=N^{2}$ of subdomains and $n$ of elements per edge ( $n^{2}$ elements per subdomain).

\begin{tabular}{c|cccc} 
& $n=5$ & $n=10$ & $n=20$ & $n=40$ \\
$K=N^{2}$ & \# Iter. & \# Iter. & \# Iter. & \# Iter. \\
\hline 16 & 8 & 9 & 11 & 13 \\
64 & 10 & 13 & 17 & 22 \\
144 & 11 & 15 & 20 & 26 \\
256 & 13 & 16 & 22 & 30 \\
400 & 13 & 17 & 23 & 32 \\
576 & 13 & 17 & 24 & 33 \\
784 & 14 & 18 & 25 & 33 \\
1024 & 14 & 18 & 25 & 33 \\
\hline
\end{tabular}

4.1.2. Nonconforming decomposition. We consider then the case of a truly nonconforming decomposition, without stabilization (formulation (10)). In our test for $e_{i}=\partial \Omega_{k} \cap \partial \Omega_{\ell}$, we set $L_{i}=\min \left\{n_{k}, n_{\ell}\right\}$. This discretization does not in general satisfy the assumptions required for the uniform stability of the discrete problem. Nevertheless, using a standard technique, it is not difficult to prove that (12) holds provided that for all $\ell, k$ such that $\left|\partial \Omega_{\ell} \cap \partial \Omega_{k}\right|>0$ it either holds that $n_{\ell}=n_{k}$ or $\max \left\{n_{\ell}, n_{k}\right\} / \min \left\{n_{\ell}, n_{k}\right\} \geq C$ for some constant $C>1$.

We considered two test configurations, both on a decomposition of $\Omega$ into $5 \times 5$ subdomains. In the first case (see Figure 1, top), for all subdomains except two, we set $n_{k}=10$, while on the remaining two subdomains (subdomains 13 and 14 , if we number all subdomains row-wise) we set $n_{k}=n$, where $n$ varies in the set $[10, \ldots, 52]$. With the above criterion used to fix $L_{i}$, we have that $L_{i}=10$ for all edges $e_{i}$, except the common edge $\Gamma_{13} \cap \Gamma_{14}$ (the two subdomains are adjacent), where $L_{i}=\max \left\{n_{13}, n_{14}\right\}=n$ varies. 


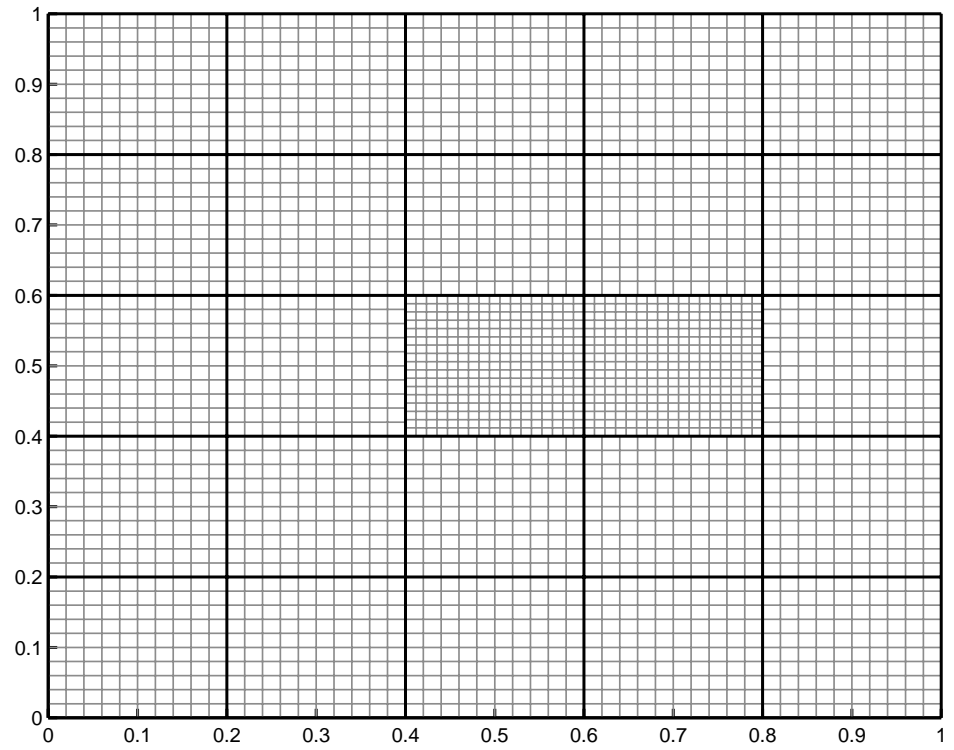

(1)

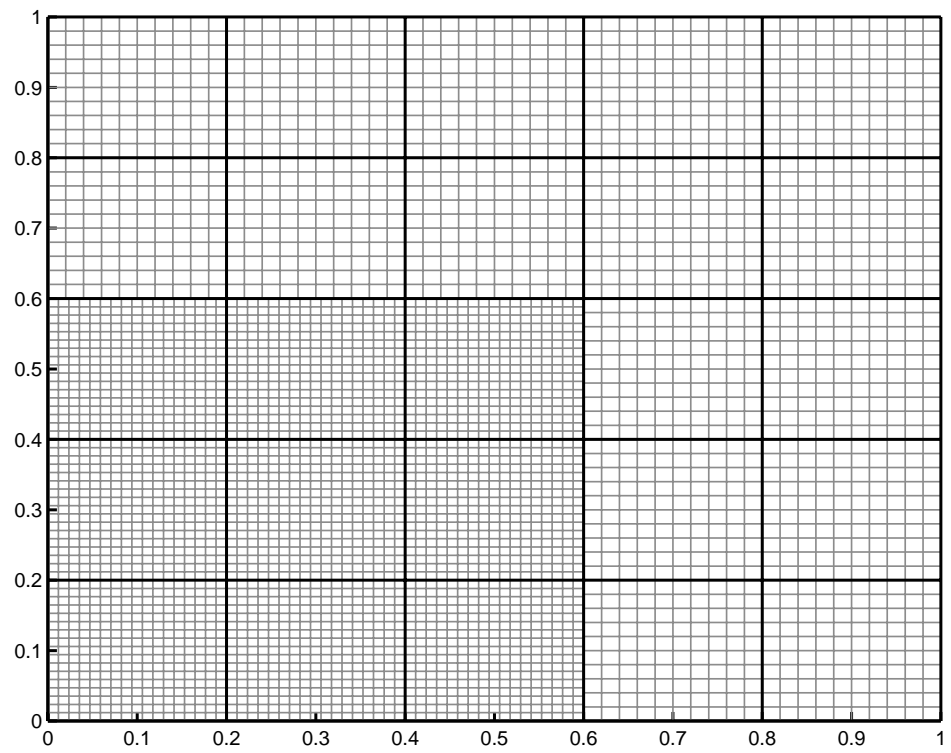

(2)

FiguRE 1. The two configurations on which the preconditioner is tested for the plain formulation (8) in the nonconforming case.

In the second configuration (see Figure 1, bottom) for all subdomains we again set $n_{k}=10$, this time except that on a three-by-three block of subdomains where $n_{k}=n$, with $n$ varying again between 10 and 52. Here, for 12 out of 40 edges of $\Sigma$, we have that $L_{i}=n$. For both configurations we tested both coarse preconditioners. The results are provided in Table 3 . 
TABLE 3. Number of conjugate gradient iterations needed for reducing the residual of a factor $10^{-5}$ for Configurations 1 and 2 (corresponding, respectively, to the top and to the bottom decompositions illustrated in Figure 1). Both coarse preconditioners (67) (BPS) and (68) (Laplace) are tested for different values of the number $n$ of elements per edge in the refined part of the skeleton (one edge for Configuration 1 and twelve edges for Configuration 2). The number of elements per edge in the remaining part of the skeleton is set to 10 .

\begin{tabular}{c|cc} 
& $\begin{array}{c}\text { Laplace } \\
\text { \# It. }\end{array}$ & $\begin{array}{c}\text { BPS } \\
\text { \# It. }\end{array}$ \\
\hline$n=10$ & 12 & 8 \\
$n=17$ & 18 & 11 \\
$n=24$ & 18 & 13 \\
$n=31$ & 18 & 13 \\
$n=38$ & 18 & 14 \\
$n=45$ & 19 & 14 \\
$n=52$ & 19 & 14 \\
\hline
\end{tabular}

(a) Configuration 1.

\begin{tabular}{c|cc} 
& $\begin{array}{c}\text { Laplace } \\
\text { \# It. }\end{array}$ & $\begin{array}{c}\text { BPS } \\
\text { \# It. }\end{array}$ \\
\hline$n=10$ & 12 & 8 \\
$n=17$ & 20 & 14 \\
$n=24$ & 23 & 16 \\
$n=31$ & 24 & 18 \\
$n=38$ & 25 & 19 \\
$n=45$ & 26 & 20 \\
$n=52$ & 27 & 20 \\
\hline
\end{tabular}

(b) Configuration 2

As one can easily see, though the theoretical estimate on the condition number only depends on $\max _{k} H_{k} / h_{k}$, which takes the same value for both configurations, in the first case in which the overall discretization of the skeleton is sensibly coarserthe mesh being fine only in a small portion (in our case one) of the edges composing $\Sigma$ - the influence of refining the mesh is weaker than in the second case, in which a relevant portion of the skeleton is refined.

4.2. Stabilized formulation. We now consider the stabilized formulation (19). Since the $H^{1 / 2}\left(\partial \Omega_{k}\right)$ seminorm is invariant under changes of scale, we can describe the stabilizing form for subdomains scaled in such a way that $\left|\partial \Omega_{k}\right|=1$ (that is, $H=1 / 4$ ). We can then identify $\partial \Omega_{k}$ with the circle $T$ of unitary length. Following the proposal of [7], the bilinear forms $[\cdot, \cdot]_{\frac{1}{2}}, k$ are designed by means of a wavelet decomposition. More precisely, we restrict the number $L_{i}$ of elements of the $i$-th edge $e_{i}$ to be a power of two,

$$
L_{i}=2^{j_{i}} \text { for some } j_{i} \geq 1,
$$

so that for all $k,\left.\Phi_{h}\right|_{\partial \Omega_{k}} \subset V_{j_{k}+2}$ where for $j>0, V_{j}$ denotes the space of $\mathbb{P}_{1}$ finite elements on the uniform grid $\mathcal{G}_{j}$ of $T$ with mesh size $1 / 2^{j}$.

The sequence $\left\{V_{j}\right\}_{j \geq 0}$ forms a so-called multiresolution analysis of $L^{2}(T)$ and it is well known (see for example [15]) that there exist several wavelet bases associated with such a multiresolution analysis. More precisely there exist several $\mathbb{P}_{1}$ compactly supported functions $\vartheta \in C^{0}(\mathbb{R})$ defined on the uniform grid of mesh size 1 and integer nodes, such that, setting

$$
\vartheta_{m \ell}=\sum_{n=-\infty}^{+\infty} 2^{m / 2} \vartheta\left(2^{m}(x-n)-\ell\right),
$$


all functions $\eta_{j} \in V_{j}$ can be written as

$$
\eta_{j}=\eta_{0}+\sum_{m=0}^{j-1} \sum_{\ell=1}^{2^{m}} \eta_{m \ell} \vartheta_{m \ell}, \quad \eta_{0} \text { constant }
$$

and such that

$$
\left|\eta_{j}\right|_{H^{1 / 2}\left(\partial \Omega_{k}\right)}^{2} \simeq \sum_{m=0}^{j-1} \sum_{\ell=1}^{2^{m}} 2^{m}\left|\eta_{m \ell}\right|^{2}
$$

Given the values of $\eta_{j}$ at the nodes of the uniform grid, the coefficients $\left(\eta_{m \ell}\right)_{m, l}$ can be retrieved by a fast $\left(O\left(2^{j}\right)\right)$ wavelet transform (FWT).

If we denote by $\mathbf{P}_{k}: L^{2}(T) \rightarrow V_{j_{k}+2}$ the $L^{2}(T)$ orthogonal projection, for $\zeta, \xi \in$ $L^{2}(T)$ we can define corresponding wavelet coefficients $\check{\zeta}_{m \ell}$ and $\check{\xi}_{m \ell}$ such that

$$
\mathbf{P}_{k}(\zeta)=\zeta_{0}+\sum_{m=0}^{j_{k}} \sum_{\ell=1}^{2^{m}} \check{\zeta}_{m \ell} \vartheta_{m \ell}, \quad \mathbf{P}_{k}(\xi)=\xi_{0}+\sum_{m=0}^{j_{k}} \sum_{\ell=1}^{2^{m}} \check{\xi}_{m \ell} \vartheta_{m \ell}
$$

Then we define the bilinear form $[\cdot, \cdot]_{\frac{1}{2}, k}$ as

$$
[\zeta, \xi]_{\frac{1}{2}, k}=\sum_{m=0}^{j_{k}} \sum_{\ell=1}^{2^{m}} 2^{m} \check{\zeta}_{m \ell} \check{\xi}_{m \ell} .
$$

It is possible to prove ([7, 14]) that the bilinear forms thus defined satisfy assumptions (A4) and (18).

Remark 4.1. Note that the coefficients $\check{\zeta}_{m \ell}$ and $\check{\xi}_{m \ell}$ are not the classical wavelet coefficients of the functions $\zeta$ and $\xi$. These would in fact be defined as the scalar product of, respectively, $\zeta$ and $\xi$ with suitable dual functions $\tilde{\vartheta}_{m \ell} \in L^{2}(T)$. The coefficients $\check{\zeta}_{m \ell}$ and $\check{\xi}_{m \ell}$ are rather the scalar products of the dual functions $\tilde{\vartheta}_{m \ell}$ with the projections of $\zeta$ and $\xi$ onto $V_{j_{k}+2}$, respectively. Naturally, such coefficients coincide with the classical ones, provided $f \in V_{j_{k}+2}$.

With this definition, the computation of $\left[u_{h}^{k}-\varphi_{h}, v_{h}^{k}-\psi_{h}\right]_{\frac{1}{2}, k}$ essentially reduces to first computing the nodal values of $\mathbf{P}_{k}\left(u_{h}^{k}\right), \mathbf{P}_{k}\left(v_{h}^{k}\right), \mathbf{P}_{k}\left(\varphi_{h}\right)$ and $\mathbf{P}_{k}\left(\psi_{h}\right)$, respectively, and then applying a FWT. As far as $\varphi_{h}$ and $\psi_{h}$ are concerned, the choice of $j_{k}$ implies that $\varphi_{h}, \psi_{h} \in V_{j_{k}+2}$ and then their nodal values are simply computed by interpolation. As far as $u_{h}^{k}$ and $v_{h}^{k}$ are concerned, we need to compute their $L^{2}$ projection on $V_{j_{k}+2}$. Observing that the mass matrix in $V_{j_{k}+2}$ has a circulant structure with bandwidth equal to three, this can also be done in $O\left(2^{j_{k}}\right)$ operations by a modification of the Croute reduction algorithm for solving linear systems with tridiagonal matrices. For further details on the implementation of the stabilized formulation we refer to 8 .

We tested the preconditioner (with both form (67) and (68) of the coarse bilinear form) on four different splittings of $\Omega$ into, respectively, $4 \times 4,8 \times 8,12 \times 12$ and $16 \times 16$ subdomains. In each case we randomly assigned the values of $n_{k}$ in such a way that for $\sim 1 / 3$ of the subdomains $n_{k}=5$, for about another third $n_{k}=10$, and for the remaining subdomains $n_{k}=15$. The four configurations considered are shown in Figure 2, For approximating the interface function $\varphi$, we used a uniform discretization of the interface $\left(L_{i}=n\right.$ for all $\left.i\right)$. For each configuration, we tested the preconditioner for different values of $n$ and of the stabilization parameter $\gamma$. The function $\vartheta$ appearing in the definition of the stabilizing bilinear form is chosen 


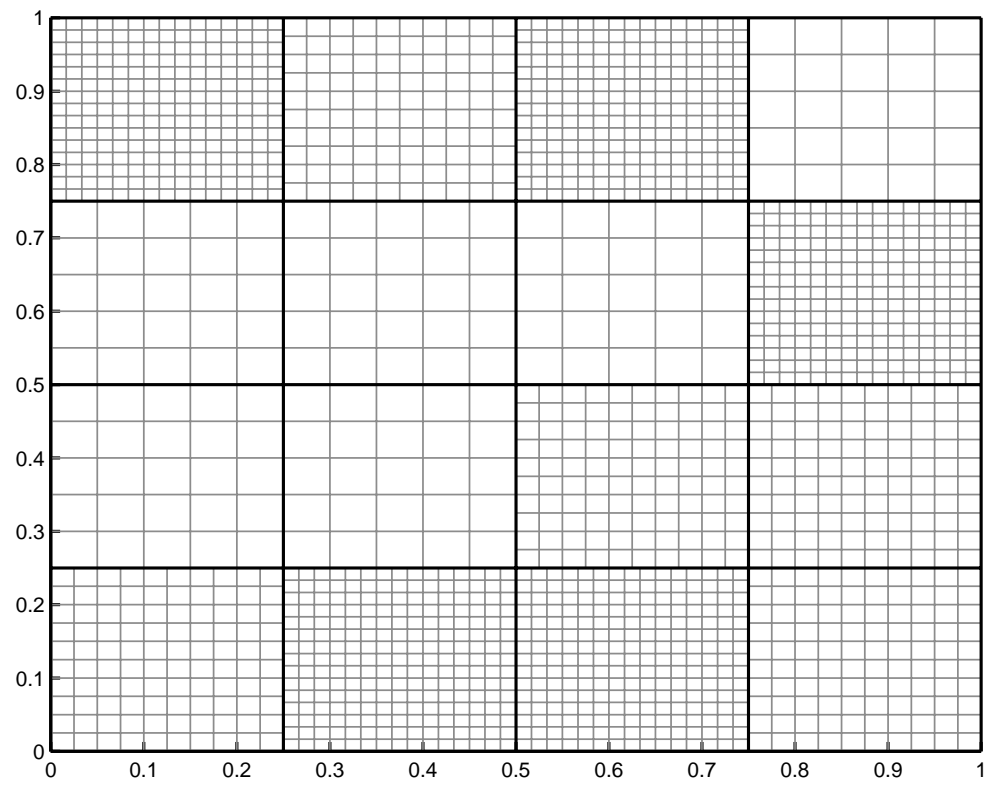

(a)

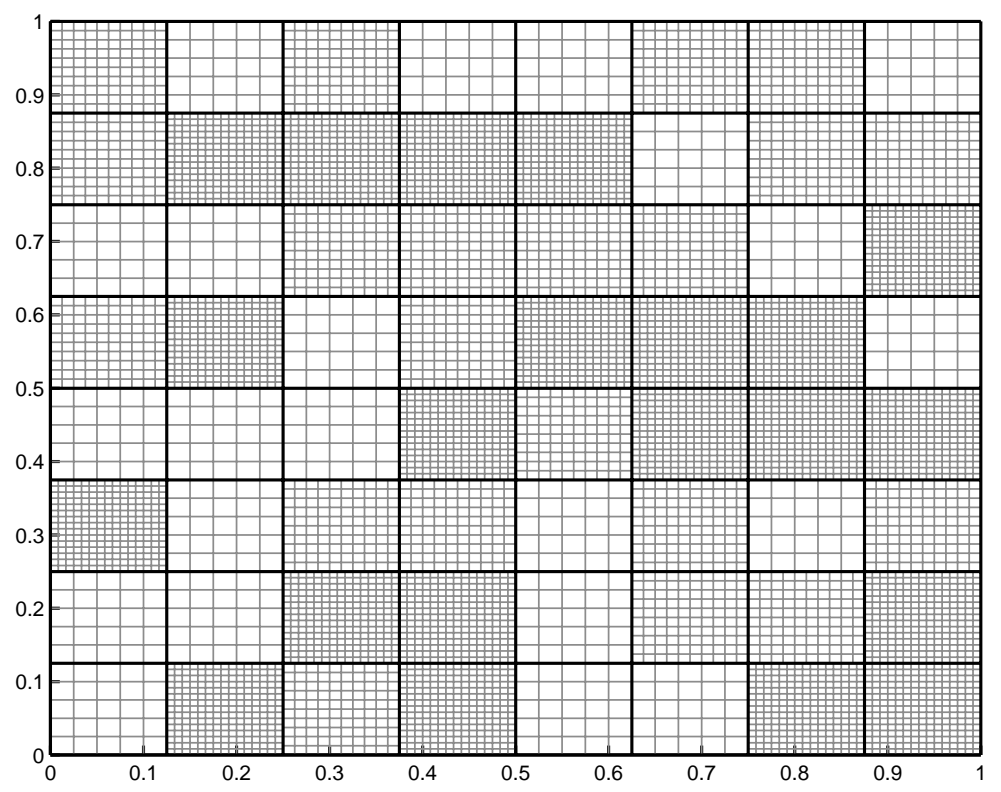

(b)

Figure 2. The four configurations on which the preconditioner is tested for the stabilized formulation (10). (Continues)

to be the piecewise linear wavelet corresponding to the $2.2 \mathrm{~B}$-spline biorthogonal setting ([15]). Note (see Table 4) that for $\gamma=0$ (no stabilization!) if $n$ is big, the CG algorithm does not converge within the maximum number of iterations (which was set equals to 100), confirming the need for using some kind of stabilization. 


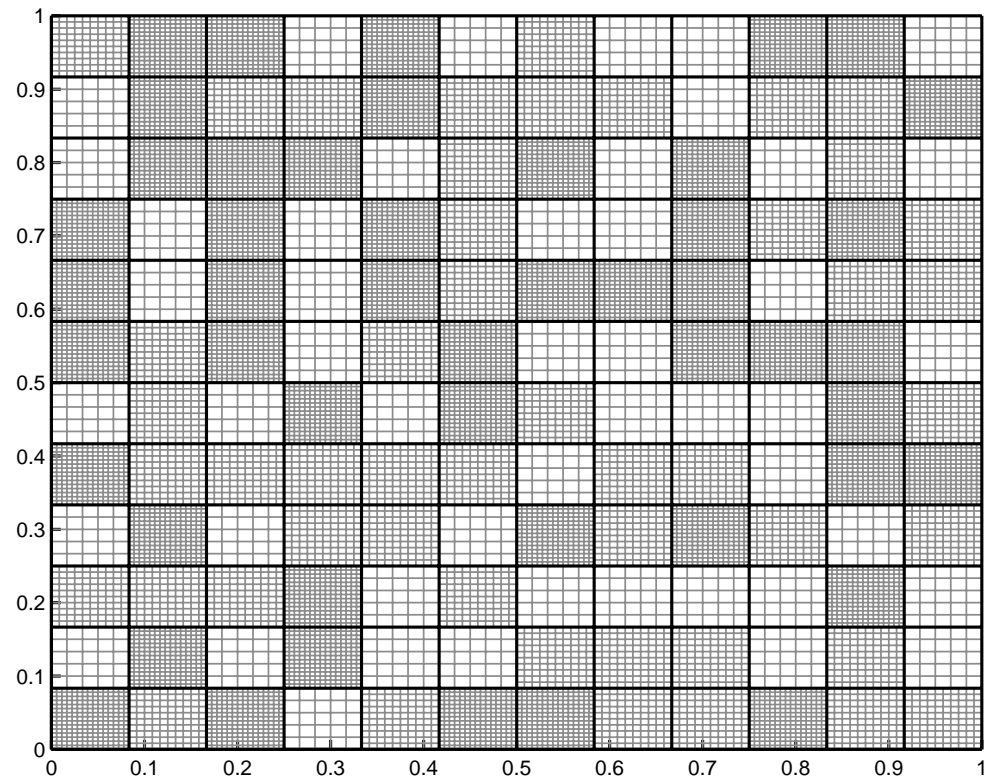

(c)

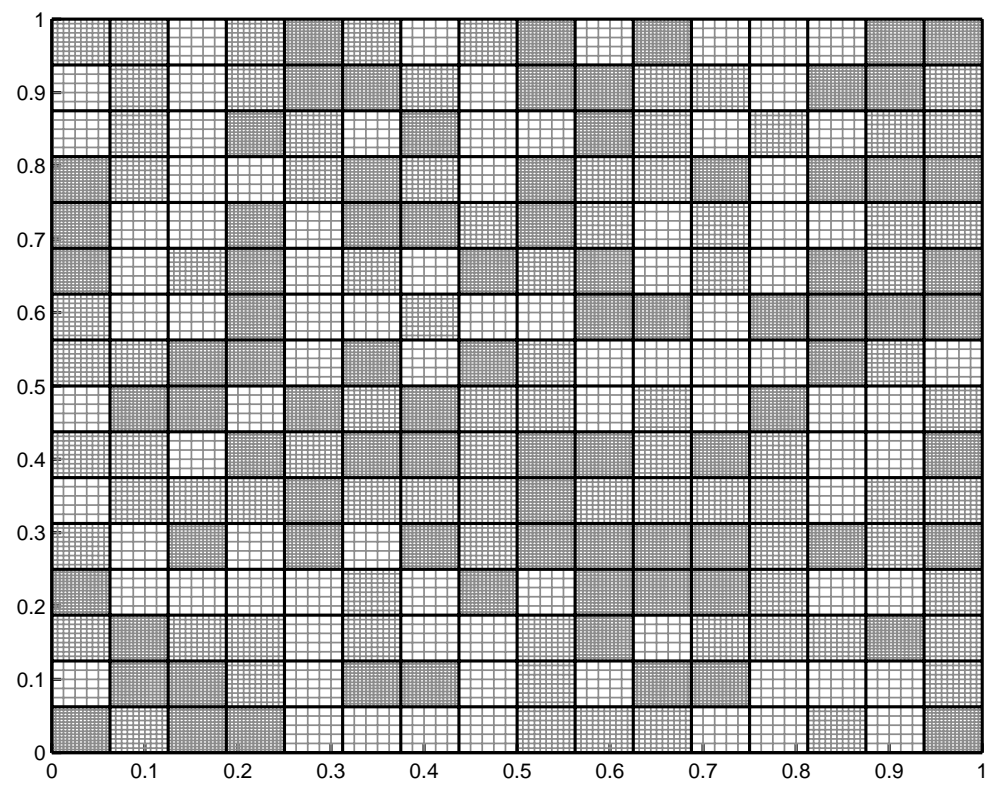

(d)

Figure 2. (continued)

As one can see from the results, the value of the parameter $\gamma$ has an influence on the conditioning of the matrix $\breve{S}^{-1} S$. If $\gamma$ is too small (see Table 5 ), the action of the stabilization term is not sufficient to compensate for the lack of validity of the infsup condition, and therefore the system behaves almost like the one obtained using the plain formulation. By increasing $\gamma$ (see Tables [6] and (7), the situation improves 
dramatically and one can see that, for instance, for $\gamma=.05$ in the case of $16 \times 16$ subdomains with $n=32$ (which corresponds to 15360 degrees of freedom for $\varphi_{h}$ ) the residual is reduced of a factor $10^{-5}$ in about 35 iterations, which is only slightly worse than the result obtained for the analogous values in the conforming case. However, when $\gamma$ is further increased (see Table 8), the performances deteriorate. This (as well as the difference with respect to the conforming case) is probably due to the fact that the constants in the estimates (26) - which have an influence on the bound on the condition number - depend on the ratio $B / b(B$ and $b$ being the constants in (18) and in assumption (A3)( $\left.\mathrm{c}^{\prime}\right)$, respectively) amplified by the factor $\gamma$. If such a ratio (which is always greater than or equal to one) in not close enough to 1 , the choice of the right balance between the original bilinear form and the stabilizing term can become important from the point of view of the spectral properties of the operator, which are essential for preconditioning.

TABLE 4. Number of conjugate gradient iterations needed to reduce the residual of a factor $10^{-5}$ on the four configurations depicted in Figure 2 with the plain formulation (10). Both coarse preconditioners are tested for different values of the number $n$ of elements per edge. As one can see for $n \geq 16$, the CG method does not converge within the maximum number of iteration $(=100)$, confirming the instability of the plain formulation for the discretization considered.

\begin{tabular}{c|cccc} 
& $($ a) & (b) & (c) & (d) \\
$n$ & \# It. & \# It. & \# It. & \# It. \\
\hline 4 & 14 & 17 & 18 & 19 \\
8 & 47 & 57 & 60 & 69 \\
16 & - & - & - & - \\
32 & - & - & - & - \\
\hline \multicolumn{4}{c}{ Laplace }
\end{tabular}

\begin{tabular}{c|cccc} 
& $($ a) & (b) & (c) & (d) \\
$n$ & \# It. & \# It. & \# It. & \# It. \\
\hline 4 & 11 & 13 & 14 & 15 \\
8 & 40 & 52 & 54 & 64 \\
16 & - & - & - & - \\
32 & - & - & - & - \\
\hline \multicolumn{4}{c}{ BPS }
\end{tabular}

TABle 5. Number of conjugate gradient iterations needed to reduce the residual of a factor $10^{-5}$ on the four configurations depicted in Figure 2 with the stabilized formulation (19) for $\gamma=$ .00125. Both coarse preconditioners are tested for different values of the number $n$ of elements per edge. One can clearly see that the method converges very badly or does not converge. This is due to the fact that, since the stabilization parameter is very small, the action of the stabilization term is not sufficient to compensate for the lack of validity of the inf-sup condition, and therefore the system behaves like the one obtained using the plain formulation.

\begin{tabular}{c|cccc} 
& (a) & (b) & (c) & (d) \\
$n$ & \# It. & \# It. & \# It. & \# It. \\
\hline 4 & 14 & 17 & 18 & 19 \\
8 & 52 & 60 & 64 & 83 \\
16 & 86 & - & - & - \\
32 & 96 & - & - & - \\
\hline
\end{tabular}

\begin{tabular}{c|cccc} 
& $($ a) & (b) & (c) & (d) \\
$n$ & \# It. & \# It. & \# It. & \# It. \\
\hline 4 & 11 & 13 & 14 & 15 \\
8 & 39 & 51 & 57 & 77 \\
16 & 69 & 92 & - & - \\
32 & 85 & - & - & - \\
\hline \multicolumn{4}{|c}{ BPS }
\end{tabular}


TABLE 6. Number of conjugate gradient iterations needed to reduce the residual of a factor $10^{-5}$ on the four configurations depicted in Figure 2 with the stabilized formulation (19) for $\gamma=1$. Both coarse preconditioners are tested for different values of the number $n$ of elements per edge.

\begin{tabular}{c|cccc} 
& $($ a $)$ & $($ b) & (c) & (d) \\
$n$ & \# It. & \# It. & \# It. & \# It. \\
\hline 4 & 14 & 17 & 18 & 19 \\
8 & 22 & 26 & 27 & 29 \\
16 & 28 & 34 & 36 & 38 \\
32 & 34 & 42 & 44 & 45 \\
\hline \multicolumn{5}{|c}{ Laplace }
\end{tabular}

\begin{tabular}{c|cccc} 
& $($ a $)$ & $($ b) & $($ c $)$ & $($ d) \\
$n$ & \# It. & \# It. & \# It. & \# It. \\
\hline 4 & 11 & 13 & 14 & 15 \\
8 & 18 & 21 & 24 & 27 \\
16 & 23 & 31 & 35 & 38 \\
32 & 30 & 41 & 46 & 51 \\
\hline \multicolumn{4}{|c}{ BPS }
\end{tabular}

TABLE 7. Number of conjugate gradient iterations needed to reduce the residual of a factor $10^{-5}$ on the four configurations depicted in Figure 2 with the stabilized formulation (19) for $\gamma=5$. Both coarse preconditioners are tested for different values of the number $n$ of elements per edge.

\begin{tabular}{c|cccc} 
& $($ a) & (b) & $($ c) & $($ d) \\
$n$ & \# It. & \# It. & \# It. & \# It. \\
\hline 4 & 14 & 17 & 18 & 19 \\
8 & 19 & 22 & 23 & 25 \\
16 & 22 & 28 & 29 & 31 \\
32 & 24 & 31 & 32 & 34 \\
\hline \multicolumn{5}{|c}{ Laplace }
\end{tabular}

\begin{tabular}{c|cccc} 
& $($ a $)$ & (b) & (c) & $($ d) \\
$n$ & \# It. & \# It. & \# It. & \# It. \\
\hline 4 & 11 & 13 & 14 & 15 \\
8 & 15 & 17 & 19 & 22 \\
16 & 16 & 23 & 26 & 29 \\
32 & 19 & 27 & 30 & 35 \\
\hline \multicolumn{4}{|c}{ BPS }
\end{tabular}

TABLE 8. Number of conjugate gradient iterations needed to reduce the residual of a factor $10^{-5}$ on the four configurations depicted in Figure 2 with the stabilized formulation (19) for $\gamma=20$. Both coarse preconditioners are tested for different values of the number $n$ of elements per edge.

\begin{tabular}{c|cccc} 
& $($ a $)$ & $($ b) & $($ c) & $($ d $)$ \\
$n$ & \# It. & \# It. & \# It. & \# It. \\
\hline 4 & 14 & 17 & 18 & 19 \\
8 & 25 & 26 & 27 & 30 \\
16 & 31 & 37 & 38 & 41 \\
32 & 35 & 41 & 43 & 48 \\
\hline \multicolumn{5}{|c}{ Laplace }
\end{tabular}

\begin{tabular}{c|cccc} 
& $($ a $)$ & $($ b) & (c) & (d) \\
$n$ & \# It. & \# It. & \# It. & \# It. \\
\hline 4 & 12 & 13 & 14 & 15 \\
8 & 23 & 24 & 24 & 27 \\
16 & 28 & 35 & 35 & 37 \\
32 & 34 & 40 & 39 & 41 \\
\hline \multicolumn{4}{|c}{ BPS }
\end{tabular}

\section{Appendix A: Some Sobolev space injection bounds}

The aim of this section is to recall some known bounds for which we provide a new proof based on the use of equivalent norms expressed through wavelet coefficients (see for instance [15]). Such proof will easily reveal the dependence of the 
constants in the bounds on the smoothness of the spaces considered and the way these constants explode as the smoothness index approaches the limit of validity of the bounds.

The key ingredient of the following arguments is the possibility of constructing two orthonormal bases

$$
\begin{aligned}
\mathcal{B} & =\left\{\rho_{k}, k=1, \ldots, 2^{j_{0}}\right\} \cup\left\{\vartheta_{j, k}, j \geq j_{0}, k=1, \ldots, 2^{j}\right\}, \\
\mathcal{B}^{0} & =\left\{\rho_{k}^{0}, k=1, \ldots, 2^{j_{0}}\right\} \cup\left\{\vartheta_{j, k}^{0}, j \geq j_{0}, k=1, \ldots, 2^{j}\right\},
\end{aligned}
$$

for $L^{2}(0,1)$, tuned up to provide norm equivalences for Sobolev spaces, respectively, without and with homogeneous boundary conditions. In particular, such bases can be constructed in such a way that all $u \in L^{2}(0,1)$ can be written both ways as

$$
\begin{aligned}
u & =\sum_{k=1}^{2^{j_{0}}}\left\langle u, \rho_{k}\right\rangle \rho_{k}+\sum_{j=j_{0}}^{\infty} \sum_{k=1}^{2^{j}}\left\langle u, \vartheta_{j, k}\right\rangle \vartheta_{j, k}, \\
u & =\sum_{k=1}^{2^{j_{0}}}\left\langle u, \rho_{k}^{0}\right\rangle \rho_{k}^{0}+\sum_{j=j_{0}}^{\infty} \sum_{k=1}^{2^{j}}\left\langle u, \vartheta_{j, k}^{0}\right\rangle \vartheta_{j, k}^{0}
\end{aligned}
$$

and that the following norm equivalences hold for all $s$ with $0 \leq s \leq 1$ :

$$
\begin{aligned}
\|u\|_{H^{s}(0,1)} & \simeq\left(\sum_{k=1}^{2^{j_{0}}}\left|\left\langle u, \rho_{k}\right\rangle\right|^{2}+\sum_{j=j_{0}}^{\infty} \sum_{k=1}^{2^{j}} 2^{2 j s}\left|\left\langle u, \vartheta_{j, k}\right\rangle\right|^{2}\right)^{1 / 2} \\
\|u\|_{H_{0}^{s}(0,1)} & \simeq\left(\sum_{k=1}^{2^{j_{0}}}\left|\left\langle u, \rho_{k}^{0}\right\rangle\right|^{2}+\sum_{j=j_{0}}^{\infty} \sum_{k=1}^{2^{j}} 2^{2 j s}\left|\left\langle u, \vartheta_{j, k}^{0}\right\rangle\right|^{2}\right)^{1 / 2}, s \neq 1 / 2, \\
\|u\|_{H_{00}^{1 / 2}(0,1)} & \simeq\left(\sum_{k=1}^{2^{j_{0}}}\left|\left\langle u, \rho_{k}^{0}\right\rangle\right|^{2}+\sum_{j=j_{0}}^{\infty} \sum_{k=1}^{2^{j}} 2^{j}\left|\left\langle u, \vartheta_{j, k}^{0}\right\rangle\right|^{2}\right)^{1 / 2}
\end{aligned}
$$

with constants independent of $s$. Such bases are constructed in such a way that the basis functions satisfy the following properties:

W.1. For all $j \geq j_{0}$ and $k=1, \ldots, 2^{j}$ the functions $\vartheta_{j, k}$ have a certain number of vanishing moments; in particular $\vartheta_{j, k}$ has zero mean value.

W.2. The basis functions are well localized and the supports of the basis functions are properly scaled with respect to $j$ : for some $N$ fixed, satisfying $N \leq$ $2^{j_{0}-2}$, we have

$$
\begin{array}{ll}
\operatorname{supp} \vartheta_{j, k} & \left.\subset\left[(k-N) 2^{-j},(k+N) 2^{-j}\right] \cap\right] 0,1[, \\
\operatorname{supp} \vartheta_{j, k}^{0} & \left.\subset\left[(k-N) 2^{-j},(k+N) 2^{-j}\right] \cap\right] 0,1[.
\end{array}
$$

W.3. The interior functions of the two bases coincide: $\vartheta_{j, k}=\vartheta_{j, k}^{0}, \quad \forall k=$ $N, \ldots, 2^{j}-N$; in other words the two bases differ only for those elements that take into account boundary conditions.

W.4. The basis functions verify

$$
\left|\rho_{k}(x)\right| \leq C, \quad\left|\rho_{k}^{0}(x)\right| \leq C, \quad\left|\vartheta_{j, k}(x)\right| \lesssim 2^{j / 2}, \quad\left|\vartheta_{j, k}^{0}(x)\right| \lesssim 2^{j / 2} .
$$


Remark A.1. Note that the constants $N$ and $j_{0}$ (and therefore $2^{j_{0}}$ ) are fixed once for all and depend only on the choice of the wavelet basis. We will therefore consider them as an $O(1)$ as far as the estimates that we are going to prove are concerned.

The normalization estimate (72), together with property W.2 on the size of the support, trivially yields the following bound on the $L^{1}$ norm of the basis functions

$$
\left\|\vartheta_{j, k}\right\|_{L^{1}(0,1)} \lesssim 2^{-j / 2}, \quad\left\|\vartheta_{j, k}^{0}\right\|_{L^{1}(0,1)} \lesssim 2^{-j / 2}
$$

In the following we will make use of a certain number of algebraic inequalities: we start by recalling the following bound on the sum of a geometric series

$$
\sum_{j \geq 1} 2^{-a j} \lesssim \frac{1}{a}
$$

as well as the well-known boundedness property of the discrete convolution operator

$$
\sum_{j^{\prime}}\left|\sum_{j} a_{j^{\prime}-j} b_{j}\right|^{2} \lesssim\left(\sum_{j}\left|a_{j}\right|\right)^{2}\left(\sum_{j}\left|b_{j}\right|^{2}\right) .
$$

Moreover, since $N$ is a fixed constant, we have

$$
\left(\sum_{k=1}^{N} a_{k}\right)^{2} \lesssim \sum_{k=1}^{N}\left|a_{k}\right|^{2}
$$

The first bound that we consider regards the injection $H^{s}(0,1) \subset L^{\infty}(0,1)$, which is known to hold for all $s>1 / 2$ [17]. As far as the dependence on $s$ of the constant in the bound is concerned, we have the following lemma.

Lemma A.1. The following bound holds for all $u \in H^{s}(0,1)$ with $\left.\left.s \in\right] 1 / 2,1\right]$ :

$$
\|u\|_{L^{\infty}(0,1)} \lesssim\|u\|_{L^{2}(0,1)}+\frac{1}{\sqrt{s-1 / 2}}|u|_{H^{s}(0,1)} .
$$

Proof. Let $u=\sum_{k=1}^{2^{j_{0}}}\left\langle u, \rho_{k}\right\rangle \rho_{k}+\sum_{j=j_{0}}^{\infty} \sum_{k=1}^{2^{j}}\left\langle u, \vartheta_{j, k}\right\rangle \vartheta_{j, k}$. Using (72) and (69), as well as properties W.1 and W.2 (which guarantees that for $x$ and $j$ fixed, $\vartheta_{j, k}(x) \neq 0$ for at most $2 N$ values of $k$ ), we have (with $\check{u}=\int_{0}^{1} u d s$ )

$$
\begin{aligned}
\sup _{x}|u(x)| & \lesssim \sup _{x}\left|\sum_{k=1}^{2^{j_{0}}}\left\langle u, \rho_{k}\right\rangle \rho_{k}(x)+\sum_{j=j_{0}}^{\infty} \sum_{k=1}^{2^{j}}\left\langle u, \vartheta_{j, k}\right\rangle \vartheta_{j, k}(x)\right| \\
& \lesssim \max _{k}\left|\left\langle u, \rho_{k}\right\rangle\right|+\sum_{j \geq j_{0}} \max _{k}\left|\left\langle u-\check{u}, \vartheta_{j, k}\right\rangle\right| 2^{s j} 2^{(1 / 2-s) j} \\
& \leq\|u\|_{L^{2}(0,1)}+\left(\sum_{j \geq j_{0}} 4^{(1 / 2-s) j}\right)^{1 / 2}\left(\sum_{j \geq j_{0}} \sum_{k=1}^{2^{j}}\left|\left\langle u-\check{u}, \vartheta_{j, k}\right\rangle\right|^{2} 2^{2 s j}\right)^{1 / 2} \\
& \lesssim\|u\|_{L^{2}(0,1)}+\frac{1}{\sqrt{s-1 / 2}}\|u-\check{u}\|_{H^{s}(0,1)} .
\end{aligned}
$$

We conclude by applying Poincaré inequality which yields $\|u-\check{u}\|_{H^{s}(0,1)} \lesssim$ $|u-\check{u}|_{H^{s}(0,1)}=|u|_{H^{s}(0,1)}$. 
The second issue that we consider is the equivalence between the spaces $H^{s}(0,1)$ and $H_{0}^{s}(0,1)$. This is known to hold for all $s \in[0,1 / 2[$ [17]. The dependence on $s$ of the constant in the bound is the object of the following lemma.

Lemma A.2. The following bound holds for all $\eta \in H^{s}, 0 \leq s<1 / 2$ :

$$
\|\eta\|_{H_{0}^{s}(0,1)} \lesssim \frac{1}{1 / 2-s}\|\eta\|_{H^{s}(0,1)} .
$$

Proof. Let $\eta \in H^{s}(0,1)$ be expanded in terms of the basis $\mathcal{B}$ :

$$
\eta=\sum_{k=1}^{2^{j_{0}}} \zeta_{k} \rho_{k}+\sum_{j \geq j_{0}} \sum_{k=1}^{2^{j}} \eta_{j, k} \vartheta_{j, k}
$$

so that

$$
\|\eta\|_{H^{s}(0,1)}^{2} \simeq \sum_{k=1}^{2^{j_{0}}}\left|\zeta_{k}\right|_{L^{2}}^{2}+\sum_{j \geq j_{0}} 2^{j} \sum_{k=1}^{2^{j}}\left|\eta_{j, k}\right|^{2} .
$$

We start by decomposing $\eta$ as the sum of four contributions:

$$
\eta=\eta_{0}+\eta^{\text {left }}+\eta^{\text {center }}+\eta^{\text {right }}
$$

with

$$
\begin{gathered}
\eta_{0}=\sum_{k=1}^{2^{j_{0}}} \zeta_{k} \rho_{k}, \quad \eta^{\text {center }}=\sum_{j \geq j_{0}} \sum_{k=N}^{2^{j}-N} \eta_{j, k} \vartheta_{j, k}=\sum_{j \geq j_{0}} \sum_{k=N}^{2^{j}-N} \eta_{j, k} \vartheta_{j, k}^{0}, \\
\eta^{l e f t}=\sum_{j \geq j_{0}} \sum_{k=1}^{N-1} \eta_{j, k} \vartheta_{j, k}, \quad \eta^{\text {right }}=\sum_{j \geq j_{0}} \sum_{k=2^{j}-N+1}^{2^{j}} \eta_{j, k} \vartheta_{j, k}
\end{gathered}
$$

and by triangular inequality we have

$$
\|\eta\|_{H_{0}^{s}(0,1)} \leq\left\|\eta_{0}\right\|_{H_{0}^{s}(0,1)}+\left\|\eta^{\text {left }}\right\|_{H_{0}^{s}(0,1)}+\left\|\eta^{\text {center }}\right\|_{H_{0}^{s}(0,1)}+\left\|\eta^{\text {right }}\right\|_{H_{0}^{s}(0,1)} .
$$

In order to bound the $H_{0}^{s}(0,1)$ norm of the four contributions, we will use the equivalent norm (70). We start by observing that for all $(j, n)$ with $n=N, \ldots, 2^{j}-$ $N$ we have $\left\langle\rho_{k}, \vartheta_{j, n}^{0}\right\rangle=\left\langle\rho_{k}, \vartheta_{j, n}\right\rangle=0$, while for the remaining $\operatorname{couples}(j, n)$ we have

$$
\left|\left\langle\rho_{k}, \vartheta_{j, n}^{0}\right\rangle\right| \leq\left\|\rho_{k}\right\|_{L^{\infty}(0,1)}\left\|\vartheta_{j, n}^{0}\right\|_{L^{1}(0,1)} \lesssim 2^{j_{0} / 2} 2^{-j / 2}
$$

using (70) and observing that for all $j$ 's the number of indexes $n$ for which $\left\langle\rho_{k}, \vartheta_{j, n}^{0}\right\rangle$ $\neq 0$ is bounded independently of $j$, we easily see that

$$
\left\|\rho_{k}\right\|_{H_{0}^{s}(0,1)}^{2} \lesssim 2^{j_{0}}\left(\left\|\rho_{k}\right\|_{L^{2}}^{2}+\sum_{j \geq j_{0}} 2^{2 j s} 2^{-j}\right) \lesssim \frac{1}{1-2 s}
$$

Using the fact that on $\mathbb{R}^{2^{j_{0}}}$ the $\ell^{2}$ and the $\ell^{1}$ norms are equivalent (the constant in the equivalence depending on $j_{0}$, which, we recall, is a fixed constant), we then easily see that

$$
\left\|\eta_{0}\right\|_{H_{0}^{s}}^{2} \lesssim \frac{1}{1-2 s}\left(\sum_{k=1}^{2^{j_{0}}}\left|\zeta_{k}\right|\right)^{2} \lesssim \frac{1}{1-2 s} \sum_{k=1}^{2^{j_{0}}}\left|\zeta_{k}\right|^{2} .
$$

We now consider $\eta^{\text {center }}$. This term contains the contribution of all functions which are interior; i.e., they do not see the boundary conditions. More precisely, 
we observe that for all $j^{\prime}, k^{\prime}$ such that $\vartheta_{j^{\prime}, k^{\prime}}=\vartheta_{j^{\prime}, k^{\prime}}^{0}$ it holds that $\left\langle\eta^{\text {center }}, \vartheta_{j^{\prime}, k^{\prime}}^{0}\right\rangle=$ $\eta_{j^{\prime}, k^{\prime}}$, while for all other $j^{\prime}, k^{\prime},\left\langle\eta^{\text {center }}, \vartheta_{j^{\prime}, k^{\prime}}^{0}\right\rangle=0$ and this yields

$$
\left\|\eta^{\text {center }}\right\|_{H_{0}^{s}(0,1)}^{2} \lesssim \sum_{j \geq j_{0}} 2^{2 s j} \sum_{k=N}^{2^{j}-N}\left|\eta_{j, k}\right|^{2}
$$

Let us now bound the $H_{0}^{s}(0,1)$ norm of $\eta^{\text {left }}$, which is the contribution of those basis functions which see the left boundary. Considering that for all $N \leq k^{\prime} \leq$ $2^{j}-N, k \leq N-1$, it holds that $\left\langle\vartheta_{j, k}, \vartheta_{j^{\prime}, k^{\prime}}^{0}\right\rangle=\left\langle\vartheta_{j, k}, \vartheta_{j^{\prime}, k^{\prime}}\right\rangle=0$ and that for $k \leq N-1$ and $k^{\prime}>2^{j}-N$ we have $\operatorname{supp} \vartheta_{j, k} \cap \operatorname{supp} \vartheta_{j^{\prime}, k^{\prime}}^{0}=\emptyset$, using (70), we can write

$$
\left\|\eta^{l e f t}\right\|_{H_{0}^{s}(0,1)}^{2} \lesssim \sum_{k^{\prime}=1}^{2^{j_{0}}}\left|\left\langle\eta^{l e f t}, \rho_{k^{\prime}}^{0}\right\rangle\right|^{2}+\sum_{j^{\prime}=j_{0}}^{\infty} 2^{2 s j} \sum_{k^{\prime}=1}^{N-1}\left|\left\langle\eta^{l e f t}, \vartheta_{j^{\prime}, k^{\prime}}^{0}\right\rangle\right|^{2} .
$$

It is not difficult to realize that the first sum on the right-hand side can be bound as follows:

$$
\sum_{k^{\prime}=1}^{2^{j_{0}}}\left|\left\langle\eta^{l e f t}, \rho_{k^{\prime}}^{0}\right\rangle\right|^{2} \lesssim\left\|\eta^{l e f t}\right\|_{L^{2}(0,1)}^{2} \leq\|\eta\|_{H^{s}(0,1)} .
$$

In order to bound the second sum, let us now bound $\left|\left\langle\eta^{l e f t}, \vartheta_{j^{\prime}, k^{\prime}}^{0}\right\rangle\right|$. We observe that, thanks to (72) and (73), for all $j, k, j^{\prime}, k^{\prime}$ we can write

$$
\left|\left\langle\vartheta_{j, k}, \vartheta_{j^{\prime}, k^{\prime}}^{0}\right\rangle\right| \leq\left\|\vartheta_{j, k}\right\|_{L^{\infty}(0,1)}\left\|\vartheta_{j^{\prime}, k^{\prime}}^{0}\right\|_{L^{1}(0,1)} \lesssim 2^{\left(j-j^{\prime}\right) / 2}
$$

as well as

$$
\left|\left\langle\vartheta_{j, k}, \vartheta_{j^{\prime}, k^{\prime}}^{0}\right\rangle\right| \leq\left\|\vartheta_{j, k}\right\|_{L^{1}(0,1)}\left\|\vartheta_{j^{\prime}, k^{\prime}}^{0}\right\|_{L^{\infty}(0,1)} \lesssim 2^{\left(j^{\prime}-j\right) / 2},
$$

which yield

$$
\left|\left\langle\vartheta_{j, k}, \vartheta_{j^{\prime}, k^{\prime}}^{0}\right\rangle\right| \lesssim 2^{-\left|j-j^{\prime}\right| / 2}
$$

Then we have

$$
\left|\left\langle\eta^{l e f t}, \vartheta_{j^{\prime}, k^{\prime}}^{0}\right\rangle\right| \leq \sum_{j=j_{0}}^{\infty} \sum_{k=1}^{N-1}\left|\eta_{j, k}\right| \cdot\left|\left\langle\vartheta_{j, k}, \vartheta_{j^{\prime}, k^{\prime}}^{0}\right\rangle\right| \lesssim \sum_{j=j_{0}}^{\infty} 2^{-\left|j-j^{\prime}\right| / 2} \sum_{k=1}^{N-1}\left|\eta_{j, k}\right| .
$$

Since the right-hand side of the above estimate does not depend on $k^{\prime}$, using inequalities (74), (75) and (76), we can write

$$
\begin{aligned}
& \sum_{j^{\prime}=j_{0}}^{\infty} 2^{2 j^{\prime} s} \sum_{k^{\prime}=1}^{N-1}\left|\left\langle\eta^{l e f t}, \vartheta_{j^{\prime}, k^{\prime}}^{0}\right\rangle\right|^{2} \lesssim(N-1) \sum_{j^{\prime}=j_{0}}^{\infty} 2^{2 j^{\prime} s}\left(\sum_{j=j_{0}}^{\infty} 2^{-\left|j-j^{\prime}\right| / 2} \sum_{k=1}^{N-1}\left|\eta_{j, k}\right|\right)^{2} \\
& \quad \lesssim \sum_{j^{\prime}=j_{0}}^{\infty}\left(\sum_{j=j_{0}}^{\infty} 2^{-(1 / 2-s)\left|j-j^{\prime}\right|} \sum_{k=1}^{N-1} 2^{j s}\left|\eta_{j, k}\right|\right)^{2} \\
& \quad \lesssim\left(\sum_{j=j_{0}}^{\infty} 2^{-(1 / 2-s) j}\right)^{2}\left(\sum_{j=j_{0}}^{\infty} 2^{2 j s} \sum_{k=1}^{N-1}\left|\eta_{j, k}\right|^{2}\right)
\end{aligned}
$$


which yields

$$
\left\|\eta^{l e f t}\right\|_{H_{0}^{s}(0,1)}^{2} \lesssim \frac{1}{(1 / 2-s)^{2}}\left(\sum_{j=0}^{\infty} 2^{2 j s} \sum_{k=1}^{N}\left|\eta_{j, k}\right|^{2}\right) .
$$

Analogously, we can prove

$$
\left\|\eta^{r i g h t}\right\|_{H_{0}^{s}(0,1)}^{2} \lesssim \frac{1}{(1 / 2-s)^{2}}\left(\sum_{j=0}^{\infty} 2^{2 j s} \sum_{k=2^{j}-N+1}^{2^{j}}\left|\eta_{j, k}\right|^{2}\right) .
$$

Adding the bounds for $\left\|\eta_{0}\right\|_{H_{0}^{s}(0,1)}, \quad\left\|\eta^{\text {left }}\right\|_{H_{0}^{s}(0,1)}, \quad\left\|\eta^{\text {center }}\right\|_{H_{0}^{s}(0,1)}$ and $\left\|\eta^{\text {right }}\right\|_{H_{0}^{s}(0,1)}$, since for $s$ in $[0,1 / 2$ [ it holds that $1 \leq 1 /(1 / 2-s)$, in view of the norm equivalence (69) we obtain the thesis.

Corollary A.1. For all $\eta \in H^{1 / 2}(0,1)$ and for all $\alpha \in \mathbb{R}$ it holds, for all $\left.s \in\right] 0,1 / 2[$, that

$$
\|\eta\|_{H_{0}^{s}(0,1)} \leq \frac{1}{1 / 2-s}\|\eta-\alpha\|_{H^{1 / 2}(0,1)}+\frac{|\alpha|}{\sqrt{1 / 2-s}}
$$

Proof. Trivially it holds that

$$
\|\eta\|_{H_{0}^{s}(0,1)} \leq\|\eta-\alpha\|_{H_{0}^{s}(0,1)}+\|\alpha\|_{H_{0}^{s}(0,1)} .
$$

In view of bound (77) the only thing that needs to be proven is a bound on $\|\alpha\|_{H_{0}^{s}(0,1)}$, which can be done by direct computation, yielding the thesis.

\section{REFERENCES}

[1] Y. Achdou, Y. Maday, and O. Widlund. Substructuring preconditioners for the mortar method in dimension two. SIAM J. Numer. Anal., 36:551-580, 1999. MR 99m:65233

[2] C. Baiocchi, F. Brezzi, and D. Marini. Stabilization of Galerkin methods and application to domain decomposition. In Future Tendencies in Computer Science, Control and Applied Mathematics. 1992. MR 94g:65119

[3] C. Bernardi, Y. Maday, and A.T. Patera. A new nonconforming approach to domain decomposition: The mortar element method. In H. Brezis \& J.-L. Lions, editors, Nonlinear Partial Differential Equations and their Applications, Collège de France Seminar, volume XI of Notes Math. Ser. 299, pages 13-51. 1994. MR 95a:65201

[4] S. Bertoluzza. Analysis of a stabilized three fields domain decomposition method. Technical Report 1175, I.A.N.-C.N.R., 2000.

[5] S. Bertoluzza. Wavelet stabilization of the Lagrange multiplier method. Numer. Math., 86:128, 2000. MR 2001h:65142

[6] S. Bertoluzza, C. Canuto, and A. Tabacco. Stable discretization of convection-diffusion problems via computable negative order inner products. SINUM, 38:1034-1055, 2000. MR 2001m:65163

[7] S. Bertoluzza and A. Kunoth. Wavelet stabilization and preconditioning for domain decomposition. I.M.A. Jour. Numer. Anal., 20:533-559, 2000. MR 2001h:65156

[8] S. Bertoluzza and G. Manzini. Wavelet stabilization of the three fields domain decomposition method: Implementation and numerical tests. In preparation.

[9] J. H. Bramble, J. E. Pasciak, and A. H. Schatz. The construction of preconditioners for elliptic problems by substructuring. Math. of Comp., 47(175):103-134, 1986. MR 87m:65174

[10] J.H. Bramble, J.E. Pasciak, and P.S. Vassilevski. Computational scales of Sobolev norms with application to preconditioning. Math. Comp., 69:463-480, 2000. MR 2000k:65088

[11] F. Brezzi and M. Fortin. Mixed and Hybrid Finite Element Methods. Springer, 1991. MR 92d:65187

[12] F. Brezzi, L. Franca, D. Marini, and A. Russo. Stabilization techniques for domain decomposition methods with nonmatching grids. In Proc. IX Domain Decomposition Methods Conference. 
THE THREE FIELDS DOMAIN DECOMPOSITION METHOD

[13] F. Brezzi and D. Marini. Error estimates for the three-field formulation with bubble stabilization. Math. of Comp., 70:911-934, 2001. MR 2002b:65159

[14] F. Brezzi and D. Marini. A three-field domain decomposition method. In A. Quarteroni, J. Periaux, Y.A. Kuznetsov, and O.B. Widlund, editors, Domain Decomposition Methods in Science and Engineering, volume 157 of American Mathematical Society, Contemporary Mathematics, pages 27-34, 1994. MR 95a:65202

[15] A. Cohen. Numerical analysis of wavelet methods. In P.G. Ciarlet and J.L. Lions, editors, Handbook in Numerical Analysis, volume VII. Elsevier Science Publishers, North Holland, 2000. MR 2002c:65252

[16] W. Dahmen and A. Kunoth. Multilevel preconditioning. Numer. Math., 63:315-344, 1992. MR 93j:65065

[17] J.L. Lions and E. Magenes. Non-homogeneous Boundary Value Problems and Applications. Springer, 1972. MR 50:2670. MR 50:2671]

[18] P. Le Tallec and T. Sassi. Domain decomposition with nonmatching grids: Augmented Lagrangian approach. Math. Comp., 64:1367-1396, 1995. MR 95m:65212

Istituto di Matematica Applicata e Tecnologie Informatiche del Consiglio Nazionale delle Ricerche, V. Ferrata 1, 27100 Pavia, Italy

E-mail address: silvia.bertoluzza@imati.cnr.it

URL: http://www.imati.cnr.it/〜aivlis 